\title{
An Ontology based Decision support for Tuberculosis Management and Control in India
}

\author{
Kumar Abhishek $^{\# 1}$, M.P. Singh ${ }^{\# 2}$ \\ \# Department of CSE, NIT Patna, Patna-800005, India \\ ${ }^{1}$ kumar.abhishek@nitp.ac.in \\ 2 mps@nitp.ac.in
}

\begin{abstract}
This paper presents an ontology based formalism of existing system for TB (Tuberculosis) control and management in India that is RNTCP (Revised National TB Control Programme). India in recent years has been highest TB burden country. There are some geographical areas of India which are badly affected by TB. The formalism presented in the paper will help in further investigation /research of a new improved method in developing a decision support system for different government / NGO (NonGovernment Organization) organizations.
\end{abstract}

Keywords: Decision Support System, Sematic Web, Ontologies, Tuberculosis, Formal Ontology, reasoning

\section{INTRODUCTION}

With reference to "Global Tuberculosis report 2014: World Health Organization (WHO)" [12], "Tuberculosis (TB)" [13] has been the most "deadliest disease" [7] which may be communicated from one person to another. The statistic in [12] indicates that number of people (all over the world) that developed TB was around 9 million in the year 2013. The number of people who died from TB was around 1.5 million out of which 360000 patient were HIV positive patient [12].

India in recent year has been the number one contributor for TB incidence rate with respect to WHO [11] [12]. In 2013, total number of patient with TB incident case was between 2 to 2.3 million [12]. There has also been an increase in the incident cases for "MDR(Multi-Drug Resistant)","XDR(Extensively Drug Resistant)","TDR (Totally Drug Resistant) TB" [12]. The "Indian government" has addressed TB as a major public health issue and addresses it by establishing "National Tuberculosis control Program (NTP)" [10] in 1960 and modifying it to "Revised National TB Control Programme (RNTCP) [10]" in 1997 which implements "WHO[11]" recommended "DOTS(Direct Observed Treatment Short course)" [13][15]. Success has been achieved by "RNTCP” [15] but incident rate has also increased since 2015.

This research paper focuses on collaborating the concept of "Decision Support System (DSS)" and "Semantic Web" [1] technologies or "Web Ontology Language [3]". The DSS in recent years has been used to support decision making activities in business or organization. DSS facilitates decision makers to generate information by analysing raw data, documents and self-knowledge in solving problem and making decision.

$D S S$ in past was known as MIS (Management Information System) the term DSS became widely used since 1980. DSS includes knowledge based systems that helps in making decision and problem solving. The Taxonomy of DSS as mentioned in [20] consists of:

- Model driven DSS[6][5] - assists in decision making by analysing data and parameters provided by the user

- Data-driven DSS [6][5] — assist in decision making by retrieving and manipulating data

- Document Driver DSS [6][5]: assist in decision making by analysing unstructured document

- Knowledge Driven DSS [6] [5]: assist in actual decision making with the help of facts, rules, procedures etc.

A DSS can be used for assisting individual person or group of person and organization in making decision[5]. Last three to four years have seen a rise in collaboration of Semantic Web and DSS especially by the European institution [5]. Semantic web supports the concept of information linking, inter-operability, integration, metadata which support in intelligent DSS development [1] [5].

"Semantic Web" [1] is "web of data" that focuses on common data representation, data sharing, reuse of data, integration across platform (heterogeneous). The main focus is to make data machine readable and machine understandable. "Semantic Web" [1] technology comprises of “XML" [6], "RDF (Resource Description Framework") [7], "RDFS (Resource Description Framework Schema)" [8] and "OWL (Web Ontology Language)" [3]. "Ontologies" [3] have been recently used in information science as it supports "knowledge representation", "logic" and "inference" that is machine readable and understandable. 
There have been good numbers of research in recent years combining "Semantic Web" [1] and "DSS" in various domains. This paper adopts an "Ontology" [3] based approach for "TB" [13] management and control in order to improve $T B[13]$ incident rate and proper patient and programme management.

The rest of the paper are organized as follows, Section 2 describes about past research/work done in "Ontology" [3] based "DSS". Section 3 provides a Logical formalism for TB Control and management using Basic Formal Ontology (BFO) [36][37][38].Section 4 describes the implementation of treatment regime using Horn clause [42] and OWL-DL(Description Logic)[43] through Semantic Web Rule Mark-up Language[41]. Section 5 discusses the conclusion and future of the work presented in this paper.

\section{LITERATURE REVIEW}

This section focuses on insights of research that has been done combing DSS and "OWL (Web Ontology Language)" [3].

M. Buranarach et.al [8] proposes a knowledge based framework for management for chronic disease care. The main idea of the proposed framework was to support decision support for clinical information component in chronic care [8]. The knowledge base for the framework is integration of ontologies, patient registry and evidence based healthcare repository. Ontology has been used for knowledge acquisition and modelling. Ontology development focusses on creating structural schema for patient repositories and using Medical Subject Headings vocabulary for semantic indexing and accessing evidence based repository [8]. Application of Semantic Web technologies in form of ontologies and metadata enable semantic integration, processing and linking of knowledge resources for better knowledge management [8]. The ontology development needs to be applied to an existing health care service and must use user feedback in evaluation of knowledge base.

The authors [11] present a better clinical decision support system for improving the decision support for management, treatment and prevention by using description logic and Semantic Web Technologies. The system aims to infer disease based signs, symptom and laboratory test as formal logic [11]. The use of Ontology and Description logic helps in inferring severity of disease based on original disease and findings respectively [11].The main issue with the system was the increase in number of assertion that resulted in degradation of inference speed [11]. There is a need to improve the reasoner support for the system to improve inference speed by trying other reasoners [11].

Ceusters, Smith [12] attempts to build an automated decision support for International Psychopharmacology Algorithm Project Schizophrenia Algorithm (IPAP-SA) by analysing the algorithm from an ontology perspective [13]. The author's analysis involved cases with referent tracking and confusion. The analysis of IPAP-SA has been performed using Basic Formal Ontology (BFO) [13] theories. The BFO can be used to express all the nodes of IPAP-SA algorithm. The disease in IPAP-SA is represented using continuant entity of BFO [13]. The use of axioms and domain ontology facilitate automated reasoning and reusable ontology such the mapping of ontologies is achieved.

SSR Abidi [14] propose a CarePlan decision support framework for patient care management. CarePlan attempts to address the issue in health care knowledge management through knowledge representation and using knowledge based solutions in clinic workflow [14]. The proposed framework develops a knowledge base comprising of two set of ontology namely clinical practice guidelines (CPG) and Clinical pathways (CP) [14]. These ontologies act as evidence repository that helps in better clinical decision support system for management of patient care [14].

S Hussain etal. [15] develops a knowledge based clinical decision support system for follow-up in treatment of breast cancer. The proposed framework consisted of three entities namely (i) Knowledge Modelling, (ii) Rules for Decision Making and (iii) Rule implementation through reasoner. First focussed on modelling knowledge in form of both declarative and procedural [15]. The knowledge consists of three ontologies namely patient ontology, domain ontology of medical knowledge, a CPG ontology based on Guideline Element Model (GEM) [15]. The second entity focused on developing rule syntax for decision making [15]. The third entity implements the rule syntax using a reasoner (JENA) [15].The ontology was tested with real-life clinical cases and recommendation of ontology was evaluated by domain experts such as medical practitioners. The developed ontology is a generic ontology which can be used and tested in other domain [15].

MM Bouamrane etal. [16] propose a knowledge enabled preoperative assessment decision making system for early identification of risks of complication during or after surgery [16]. The proposed system makes use of semantic web technologies such as modular ontology and automated reasoned [16]. The knowledge base was developed using National Institute of Clinical Excellence (NICE) guideline for making a preoperative decision system [16].

Naomi Wrighton and Christopher D. Buckingham [17] propose ontology for decision support system for mental risk assessment. The ontology developed for the system was based on Galatean Risk Screening tool Data structure (GRiST) [17]. The GRiST data structure was represented by three different ontology describing the 
information in GRiST tree at logical level and physical level [17]. The developed ontology also provides tool for representing uncertainty and risk quantification [17].

M Turki etal. [18] propose an ontology for representing process of organization via a formal approach named as COOP (core ontology of organization process) [18].The COOP structure is based on three concept namely (i) Foundation Level, (ii) Collective Level and (iii) Organization Level. The first concept focuses on foundation level via descriptive ontology for linguistic and cognitive engineering (DOLCE) [19] for representing object and process as Endurants [19] and Pendurants [19] for analysis of reality [18]. The second concept focussed on collective level where Agentive Entity were used to represent Collectives [18]. Third concept dealt with organization level that focussed on deining Organization and their processes [18]. Further authors also identified new concepts that can be added to $C O O P$ as well as concept of goal and procedure have been introduced in priority for refining the model of process of organization [18].

I Turcin etal. [20] present an integration of data mining and data warehousing with domain ontology. The authors describe a domain ontology for lower limbs using Ontologie du Systeme Musculo-squelettique des Membres Inferieurs (OSSMI) ontology as the base vocabulary[20]. The data for proposed system consists of demographic and time sequence data that is collected from varied sources and pre-processed into structured format using Ontologybased ETL (Extract, Transform and load) tool which gave good results in data refinement and interpretation of results [20]. The ontology ETL tool had better results than OWL (web ontology language) [20].

Junyi Chai and James NK Liu [21] develope a system to support group decision framework with complex decision group and task [21]. The proposed system is named as ONTOGDSS (Ontology based Group Decision Support System)[21]. The ontology is first used for construction of the complex structure of group and task [21].Based on ontology model, developed framework of support system and group decision process are provided [21]. Then at the last authors presents an ontology based approach for representing decision problem and extraction [21].

The authors [22] propose a decision support system for management audit via ontology. A generic quality management system and company quality management ontology was developed [22]. Based on these ontologies, this work tries to address the problem faced in decision making in audit management such as knowledge acquisition, knowledge translation, addressing partial knowledge, collaboration among auditor and auditees [22].

HT Wand and AU Tansel [23] use the concept of composite ontology for creating a decision support system framework for medical diagnosis [23]. The proposed system has been outlined in two folds namely (i) Knowlegde Base and (ii) Reasoning [23]. The first focusses on constructing knowledge for medical diagnosis by developing a composite ontology for identifying simple pattern matching for disease based on input such as patient sign and symptoms [23]. The second step made use of case based reasoning for refining decision based on semantic similarity [23].

C.Ding etal. [24] propose a travelling decision support system for person with deficiency using Linked data principles [24]. The proposed system consisted two phase namely (i) Data Integration model and (ii) Decision support Model [24]. The first phase termed as Data Integration model [24] focussed on extraction of heterogeneous data ( raw, open and linked data) and inter-link the extracted data and publish them using Linked data principle [24]. The second phase used the Linked data generated from the Data Integration Model [24] for finding optimized travel decision support for users based on physical capabilities of the user [24]. The author has exposed the second phase for researchers for exploring the best algorithm for finding best travel decision applying principles of Linked Data nad based on user's capabilities [24].

Amin Saremi etal. [25] propose a framework $\mathrm{O}_{2}$ DSS(Ontology based Decision Support System) which exploits ontology in Decision Making and user modelling. Pervasive computing is system or environment that can easily adapt to the changing operating platform and user requirements [25]. This focusses on a decision support system that will help the pervasive system to be adaptable with help of information on context of use [25] such as location, task and user preferences. The use of ontology in representing context in data will help DSS to provide better support to the users [25]. The proposed architecture consists of five layers namely (i)Presentation Layer (ii)Meta-Model Layer (iii)Model engine Layer (iv)Cocept Layer and (v) Ontology Layer [25] The presentation layer [25] focusses on specifying problems by converting the user natural language query into description logic(DL) query. The meta -model layer [25] uses the DL query generated from presentation layer and extract meta-model concepts. This model acts as TBox (terminology axioms). The model engine layer [25] is an ABox (assertion axiom) for compatibility representation between model and solver, model taxonomy and matching of patterns. This layer makes use of the next layer i.e. concept layer [25] for retrieving concepts for execution. The concept layer [25] provides a domain ontology structure to be used by model layer [25]. The ontology layer built a knowledge base using the concept layer for representing actual state of the world [25]. 
There are many other research work based on ontology and DSS in other domain like waste water management [26][27] , environment decision support system [28] etc.

The authors [26] make an effort for improving environmental decision support system by using semantic web technology [26]. The OntoWEDSS decision support prototype is proposed for waste water management [26]. The proposed architecture is an extension of WaWO(waste water ontology) [27] by incorporating case-based and rule-based reasoning along with $\mathrm{WaWO}[26]$. The enhanced model improved information model for waste water treatment as well as reasoning. The OntoWEDSS provides better diagnostic system for determining faulty states of treatment plant via ontological cause effect-relation micro-organism $\leftrightarrow$ problematic solution and state of the plant $\leftrightarrow$ suggested action [26]. The ontology in proposed architecture improves the communication and ambiguities among agents and different elements of environmental decision support system [26].

The authors [28] propose ontology based knowledge base in DSS (decision support system) as main data Structure [28]. This semantic representation of contents helps the DSS in making decision [28]. The proposed idea has been employed in an existing environment DSS known as PESCaDO ontology for personalized environment decision support [29]. The PESCaDO DSS proved how the semantic knowledge base helped DSS in information integration, structured content processing and logical reasoning and inference [28]. This ontology was evaluated by a team of environmental experts who gave good score for completeness and appropriateness [28].

The above summary concentrates on use of Ontology in field of decision support system; apart from DSS, Semantic web and its technologies. Especially Ontologies have been frequent used in research related to knowledge enable health management systems, physical cyber system, sensor web etc. The ontologies used in this are addresses issues related to incompleteness, uncertainty, perception, software engineering, enterprise application, provenance, annotating sensor data.

The authors [30] present a nice study on application and advantages of ontology in software engineering [30]. They proposes a "Ontology Driven Architecture(ODA)" which consist of four categories namely ontology driven development (ODD), ontology -enabled Development (OED), ontology based Architecture(OBA) and Ontology enabled architectures that focussed on how ontologies can be applied in different context of software engineering [30]. The major benefits of ontologies such as integration of information from heterogeneous sources, interoperability and reuse will enhance software engineering [30].

Daniel Oberle [31] presents how the technology features of ontology benefits an enterprise application. The features such as conceptual modelling, reuse, web compliance, optimal use of information within organization and representing integrated and easy to understand view has contributed to innovation in business scenario [31]. Further author presents case studies such as FindGrid, a successful integration of ontologies with SAP whereas case studies like xCarrier and Oil Gas where the applied ontology didn't get acceptance from the users [31]. The author also suggests what possible work can be done in near future for successful integration of ontology in enterprise application [31].

Rommel Novaes Carvalho etal [32] [33] propose a probabilistic web ontology language (PR-OWL) which extends ontology to represent MEBN (Multi-entity Bayesian Network) concept and theories. MEBN, an integration of first-order logic and Bayesian probability theory, helps in reasoning under uncertainty that can be expressed using first-order logic. The PR-OWL case study has been developed to help the Brazilian Office of the Comptroller General (CGU) in fraud detection in procurement [33]. PR-OWL was also used in a case study for identifying ship of interest (suspicious) in maritime domain [34]. The proposed combination of uncertainty reasoning with Semantic Web technology will be beneficial for decision support system [33][34].

Semantic web Technologies are also used frequently today in field of Sensor Web[45], Physical Cyber System [46], Provenance management[47], proactive personalized health care [48] etc.

The main target of this paper is to provide a formalized framework for Tuberculosis (TB) control and management in India. The Government of India has its own framework based on Wold Health Organization (WHO) named as RNTCP (Revised National Tuberculosis control Programme) since 1997. Success has been reported under RNTCP implementation but still India counts as one of the major contributors of TB incidence rate in world. The current state of RNTCP is a diversified setup divided into five level namely (i) central,(ii) state, (iii)district,(iv) sub-district and (v)peripheral health institution. Each level has different actors responsible for implementing the strategy made by the government. The organizational structure of RNTCP is very complex and multi-tier, which may be a hurdle in getting information, information integration, reusing of information and proper working of the system. The proposed formalism will focus on a unified framework that will help in decision making.

The ontology formalism provides a strategy to represent information in a computer traceable form, by using concepts from information science, computers, linguistic, logic and philosophy[38]. Ontology also provides feature of conceptual modelling for easy and integrated view representation, web compliance, formality, reasoning, reuse and optimal use of information. 


\section{FORMAL ONTOLOGY BASED REPRESENTATION OF RNTCP}

This section focuses on representing RNTCP (Revised National Tuberculosis control Programme) using an upper level ontology such as Basic Formal Ontology (BFO) [36]. The main purpose for use of ontology is to provide a structure to the electronic information repositories in order to support inter-operability among the different repositories and a unified information framework for understanding [36].

BFO (provides a way to represent a general entity that is not specific to a problem domain and has been widely used in representing Bio-Medical information [35]. This paper makes use of BFO (Fig. 1) to represent RNTCP a Government of India strategy for TB Control and management based on WHO STOP TB Strategy. Basic formal ontology realizes an entity (object/resource) into two basic categories namely continuant or substantial entity and processual entities or occurrent [36]. The continuant can be defined as entities that continues to persist through the time while maintaining its identity [36]. The continuant is further sub divided into three categories namely dependent continuants, independent continuants and spatial region [36]. The occurrent in BFO are defined as the entities that can happen or occur in time [36]. The occurrent are classified into three subclasses in name of spatiotemporal region, processual entity and temporal region [36].

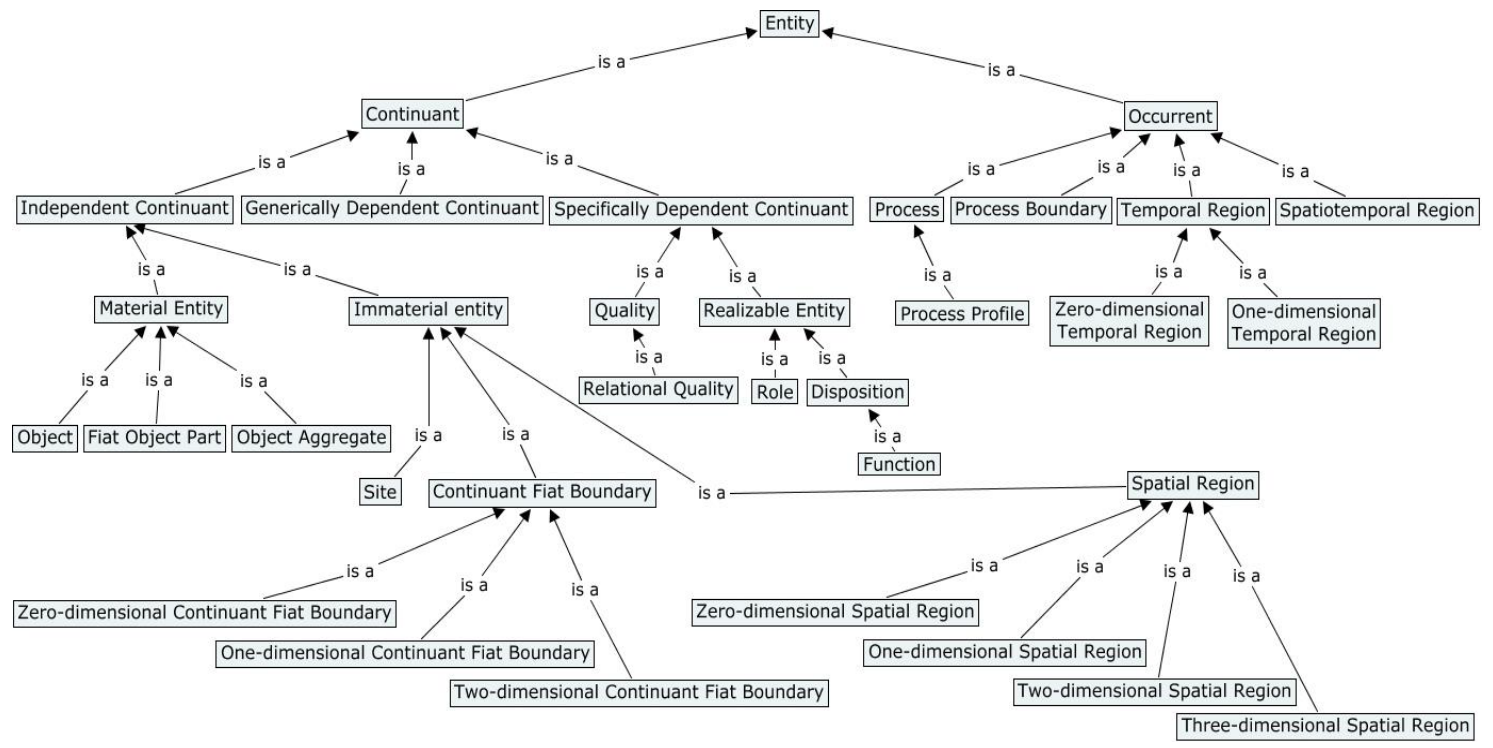

Fig. 1. Basic Formal Ontology (BFO) Hierarchy (Version 2.0) [36]

The proposed model uses the concepts of continuant and concurrent [36][37][38] to represent the RNTCP program. The organizational and operational setup of RNTCP comprises of five levels: National level, State level, district level, sub-district level and peripheral health institution level [39]. Every level comprises of actors responsible for implementing RNTCP. The National level comprises of CTD (Central TB Division), Directorate General Health Services, Ministry of Health and Family Welfare (MoHFW, Govt. of India), Joint Secretary(MoHFW), National Tuberculosis Institutes as an assistance to CTD, five Chief Medical officer(CMO) under CTD responsible for implementing and managing RNTCP program. These actors are now modelled into Basic Formal Ontology.

MoHFW (Ministry of Health and Family Welfare, Govt. of India) is an independent Government organization which undertakes many nation level activities of which RNTCP is concerned with Tuberculosis control in India. Directorate General Health Services, Ministry of Health and Family Welfare, Govt. of India (DGHS) is a specialized wing of MoHFW which has the Central TB Division to manage the RNTCP. Any organization can be realized as an aggregate whose member parts have roles of specific types. Hence, MoHFW and DGHS is realized as 'object aggregate' under Independent Continuant. DGHS is also declared as a subclass of MoHFW. The CTD is the actual location where all administrative and financial work is done and also dependent upon the DGHS or the MoHFW and hence recognized as 'site'. Its subunits are realized as its subclasses. The DDG TB is post held by a specific person hence realized as a role but at the same time it belongs to the CTD and hence also its subclass.

National Tuberculosis Institutes equipped with laboratory facilities, responsible for tuberculosis related clinical researches which are also realized as independent 'object aggregate' and includes all the three National Institutes as its 'individuals'. These individuals are independent and are of the type National TB Institute. They can also be considered as National Research Laboratory (NRL). 


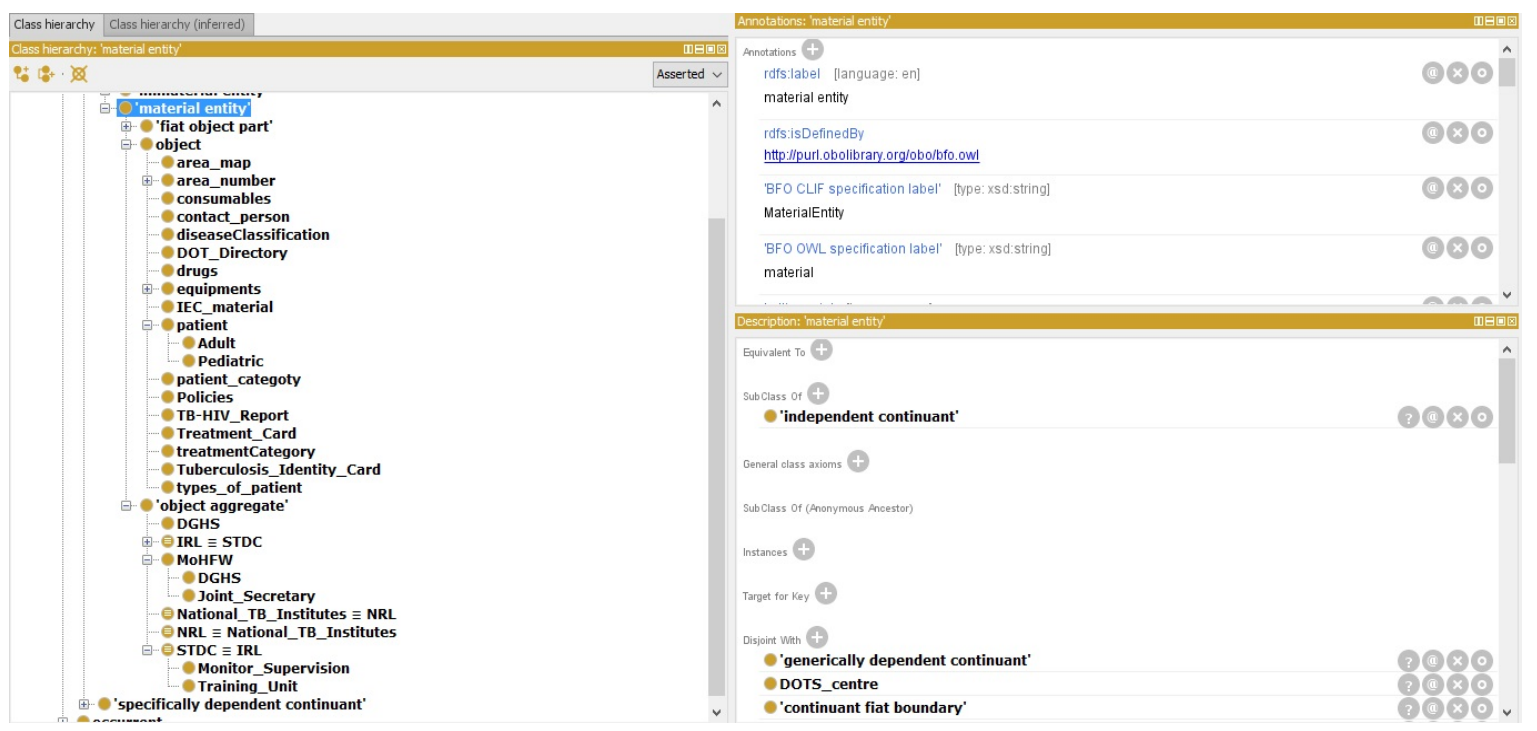

Fig. 2. Entities as Object and Object Aggregate

Fig. 2 also shows Independent Continuants categorized as immaterial entity and material entity. Immaterial entity includes 'site' which are material dependent and 'spatial region' which exist independently of material entities. DMC/PHI (Designated Microscopy center/Peripheral, Sputum Collection Centers, etc. depend upon Tuberculosis Unit hence realized as 'site'. Spatial Region includes immaterial entity like NGOs, WHO, hospitals/medical colleges, etc. which work in their respective fields independently.

The State Training and Demonstration Centre are also recognized as 'object aggregate' because they work in independent manner. State Drug Store is center for various types of medicines and drugs other than Tuberculosis also hence realized as 'site'. STDC is concerned with training and evaluation purposes and also assists STO. The "Training unit, Supervision and Monitoring unit and Intermediate Reference Laboratory (IRL)" [39] are constituent parts of STDC hence realized as the 'object aggregate' and also subclass of STDC. IRL can itself be considered as STDC under special circumstances.

The patient is considered as an 'object' entity because it holds the place of importance in our domain. A patient can simultaneously be on treatment schedule of more than one disease other than Tuberculosis.

The state Level structure of RNTCP, i.e. STC (State level TB Cell) is headed by State Tuberculosis Officer (STO). The STO is responsible for implementation of RNTCP strategies in a state as per the guidelines and technical instruction provided by the CTD. The State TB Training and Demonstration Centre (STDC) works in coincidence with the STC. The other important staffs/actor in STC involve Medical Officer, State IEC Officer, State Accountant, Secretarial Assistant, Pharmacist and Data Entry Operator.

The State TB Cell is dependent on MoHFW for finance and resources. It is the center for management of RNTCP activities at the state level. Hence STC is recognized as 'site'. Various staff of the STC are the actual actors involved at this level and hence recognized as 'role'.

Fig. 3 shows State TB Cell as a 'site'. The individual actors like STO, Accountant, etc. as 'role'. Major functions are also represented. Relationship with NGOs, IRL, etc. can also be seen.

RNCTP activities within a district will be performed and operationalized under direct influence of the Chief District Health Officer (CDHO) / Chief District Medical Officer (CDMO). The District Tuberculosis Centre (DTC) can be considered as the location for managing all the Tuberculosis control activities within the district. In a more precise way we can also say that DTC is more like a managerial workplace than a clinical workplace.

The DTC is headed by the District TB Officer (DTO). The DTO oversees various RNTCP activities at the district level in accordance with the Programme guidelines [19]. The DTO involves other sectors in RNTCP into a better compliance. The MO, Statistical Assistant and other paramedical staff provide significant assistance to the DTO. RNTCP guidelines specify that each district needs to have a trained DTO.

CDMO is dependent upon MoHFW for finance and resources. Similarly, all staff requirement is fulfilled by STDC. All DTO within state submit reports to the STO which are also forwarded to CTD. Hence DTO and CDMO belonging to district are the actors hence recognized as 'role'. DTC is the actual location where all managerial RNTCP activities at district level are performed and hence is recognized as 'site' (as shown in Fig. 4). 


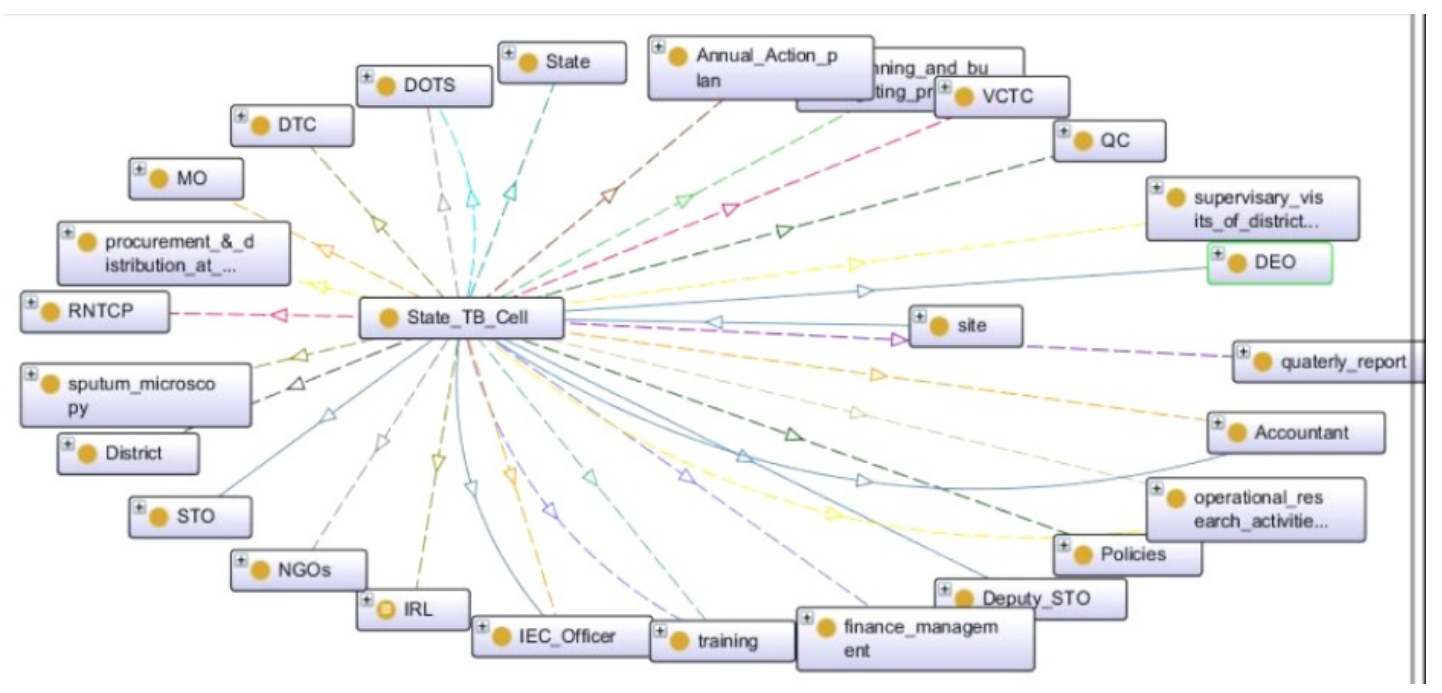

Fig. 3. State TB Cell, its actors, its functions and relation with other entities.

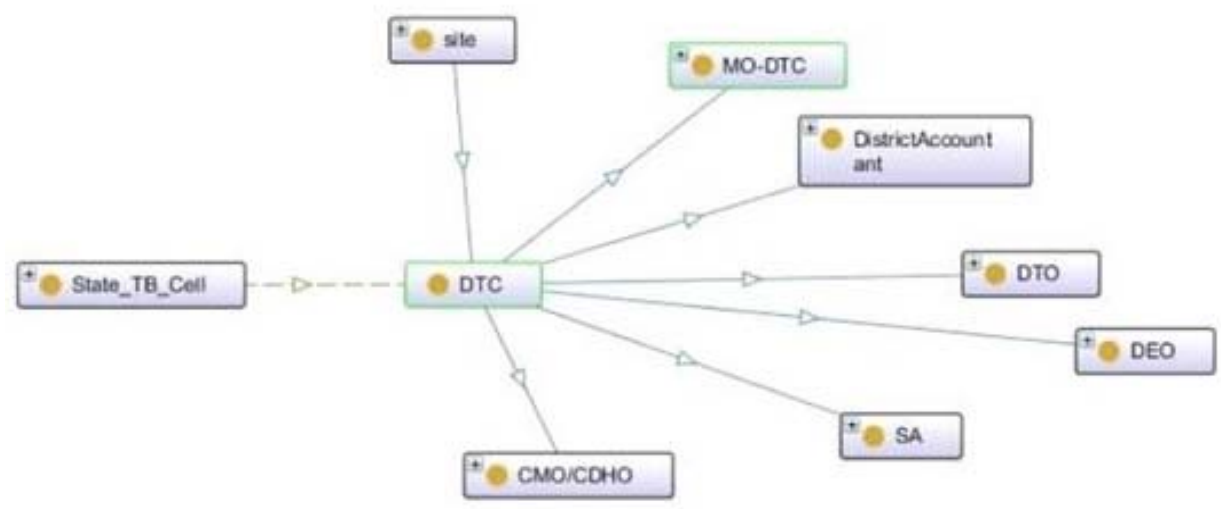

Fig. 4 DTC and its actor

The Tuberculosis Unit(TU) under DTC comprises of Medical Officer-Tuberculosis, Senior Treatment Supervisor (STS) and a Senior Tuberculosis Laboratory Supervisor (STLS) as important staff /actors. The treatment of patient and testing of suspected are performed by TU, at TU is also referred as DMC. TU acts as a nodal point for TB control activities in the sub-district.

TU is considered to be the location for maintenance of records and registers. It depends on MoHFW and STDC hence recognized as 'site'(as depicted in Fig.5). Various actors like STS, STLS, etc. which follow RNCTP guidelines and are expected to use our Decision Support System are recognized as 'role' which participate in maintenance and updating related works.

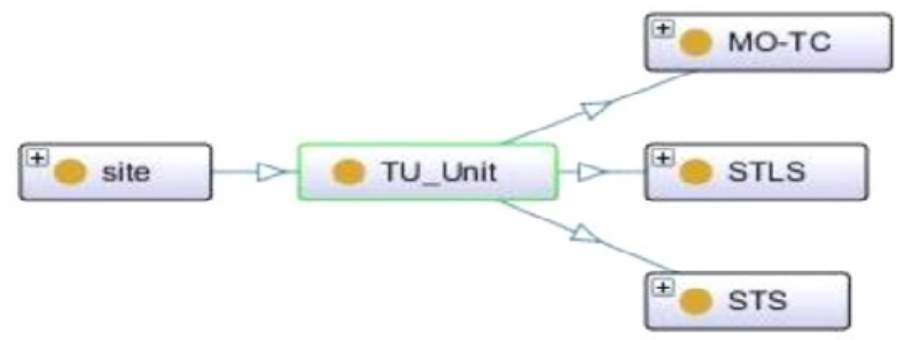

Fig. 5 TU_unit with its actors.

Fig. 6 shows the Tuberculosis Unit realized as a 'site' because it is the center for RNTCP activities at the sub-district level. It also shows actors which work at this particular level realized as roles.

The PHI (Peripheral Health Institution) within district includes PHCs, CHCs, referral hospitals, major hospitals, specialty clinics / hospitals / TB hospitals / Medical colleges. All the PHI with or without DMC needs to send a monthly report to the higher levels. 
Various kinds of PHIs are required to send reports to subsequent higher level. Government run PHIs depend upon MoHFW and STDC hence they are recognized as 'site'. These will serve as the actual location where treatment is carried out. Actors involved at these PHIs will be MO, MPW, MPHS, etc. are realized as 'role'. These actors follow RNTCP guidelines and will also be the target audience of our Decision Support System.

The Joint Secretary is the financial and administrative head of RNTCP whereas the DDG TB is the overall head of the Programme. There is a CMO designated for each unit of CTD. At state level STO is head of State TB Cell. For the purpose of Tuberculosis control a specialized District Tuberculosis Centre is present at every district headed by a DTO. SA and MO will assist the DTO. Within a district there will be Tuberculosis Unit associated with a DMC. MO-TC will head the TU and will be assisted by STS and STLS. A TU has many PHIs involved which can be public/private in nature. PHI will have staff like MO, LT, MPW, MPHS and other DOTS staff. Within a PHI there will be different units namely receiving unit and referring unit for the purpose of relocation of patient under necessary circumstances. DOTS Center acts as the place where patients get their dosage and treatment is carried on. Sputum Collection Centers are present in remote and rural areas for the purpose of sputum collection.

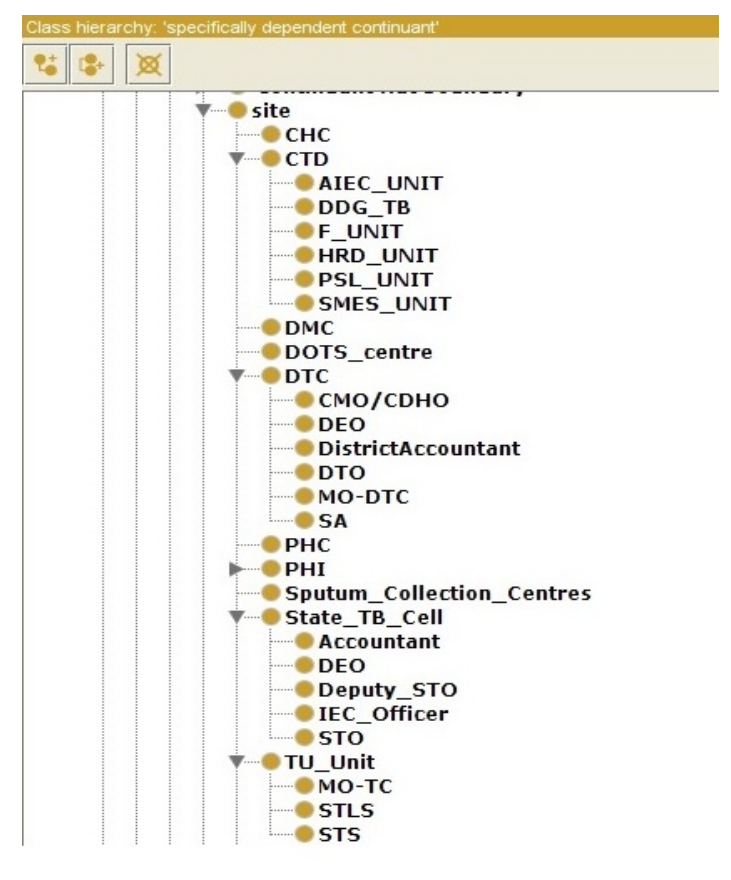

Fig. 6 Actors involved in RNTCP as 'role'

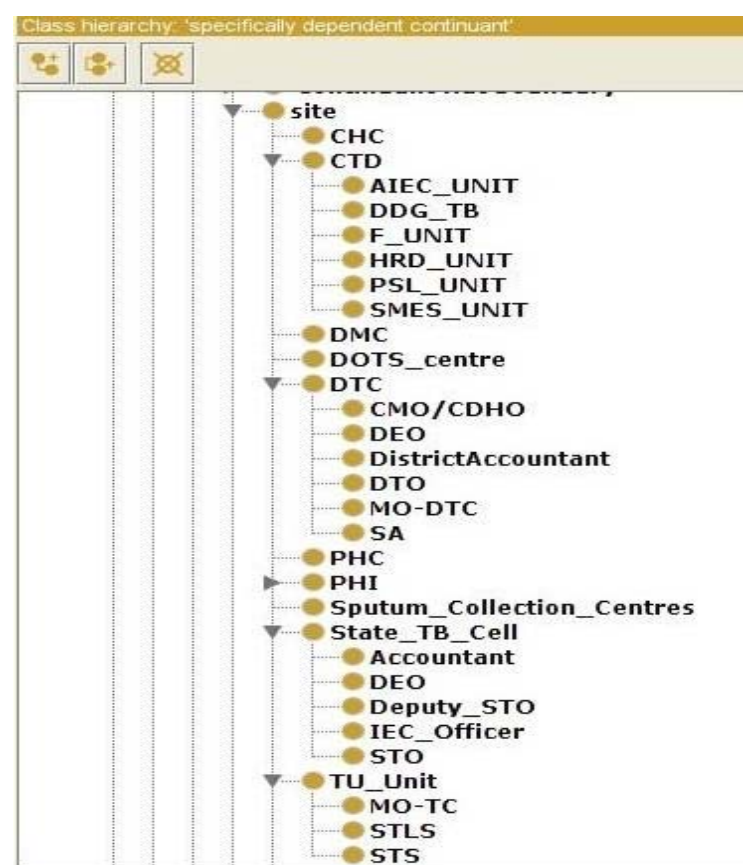

Fig. 7 Levels of RNTCP as 'site’

Fig. 7 depicts the actors who were described earlier are realized as 'role' are now seen under 'site' this happens when actors are declared as a subclass of their appropriate organizations or modules. This is due the automatic reasoning capabilities of Ontology. BFO supports transitive relation which is best explained here. If actors are subclasses as 'role' and subclasses of 'role' is declared as subclasses of 'site', then actors become subclasses of 'site' as well.

\section{A. Realization of RNTCP in $B F O$}

RNTCP is sum totality of many different processes to be performed by different actors (continuants) at different locations (site/spatial regions). Many temporal parts of RNTCP are dependent upon MoHFW and hence RNTCP is recognized as 'history' a subclass of 'process'. Process is an occurrent which has temporal parts and depends upon some material entity.

Fig. 8 shows RNTCP recognized as process, a subclass of 'history' to be more precise. RNTCP being a nationwide Programme will include other entities (occurrents) like Annual Action Plan, external review, training plans, policies, IEC strategy and guidelines, and many other activities. All these entities will unfold with time and will be associated with one or more continuants and hence they are considered as occurrent and recognized under superclass RNTCP. 


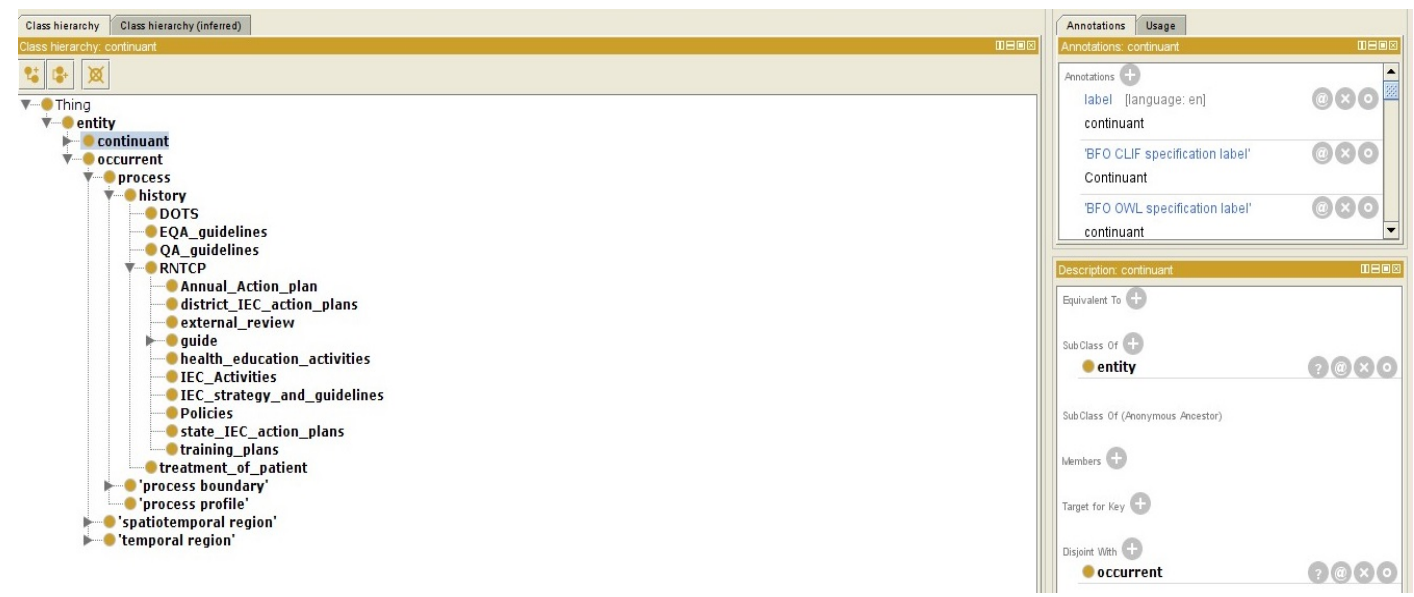

Fig. 8 RNTCP as a Process

All other occurrents that will be realized with the functions of various actors within RNTCP are recognized as 'process boundary' and 'spatiotemporal region'. The former represents a process which is a temporal part of another process and it has no proper temporal parts and the latter is an occurrent entity that is part of space-time. Some occurrents belong to 'temporal region'. These occurrent entities are part of time as defined relative to some reference frame.

DOTS is realized as a 'process' covering many processes under itself. Sputum Smear Examination is conducted at the initial diagnosis stage before actual treatment begins. Treatment or medication is itself a process covered under DOTS and hence declared as its subclass. Additionally, both the entities(processes) i.e. the test and treatment are recognized as 'process profile'. This realization is achieved because both are covered under DOTS but have nothing in common, yet we can easily say that treatment is mutually dependent upon on test.

Even though DOTS is considered as a sure cure for Tuberculosis, a person can acquire this particular disease in different forms during different stages of life.

Fig. 9 and Fig. 10 show different occurrents recognized based upon above classification.

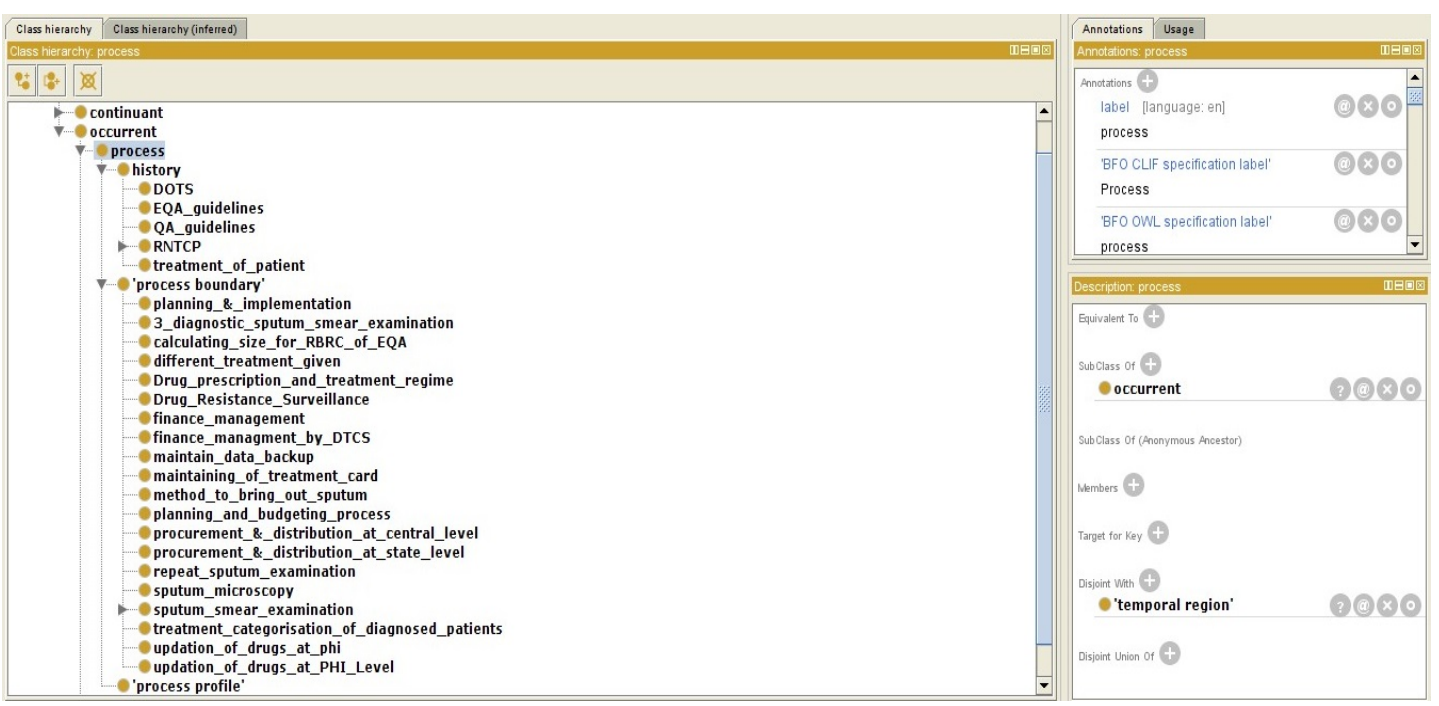

Fig. 9 Entities under Process Boundary 


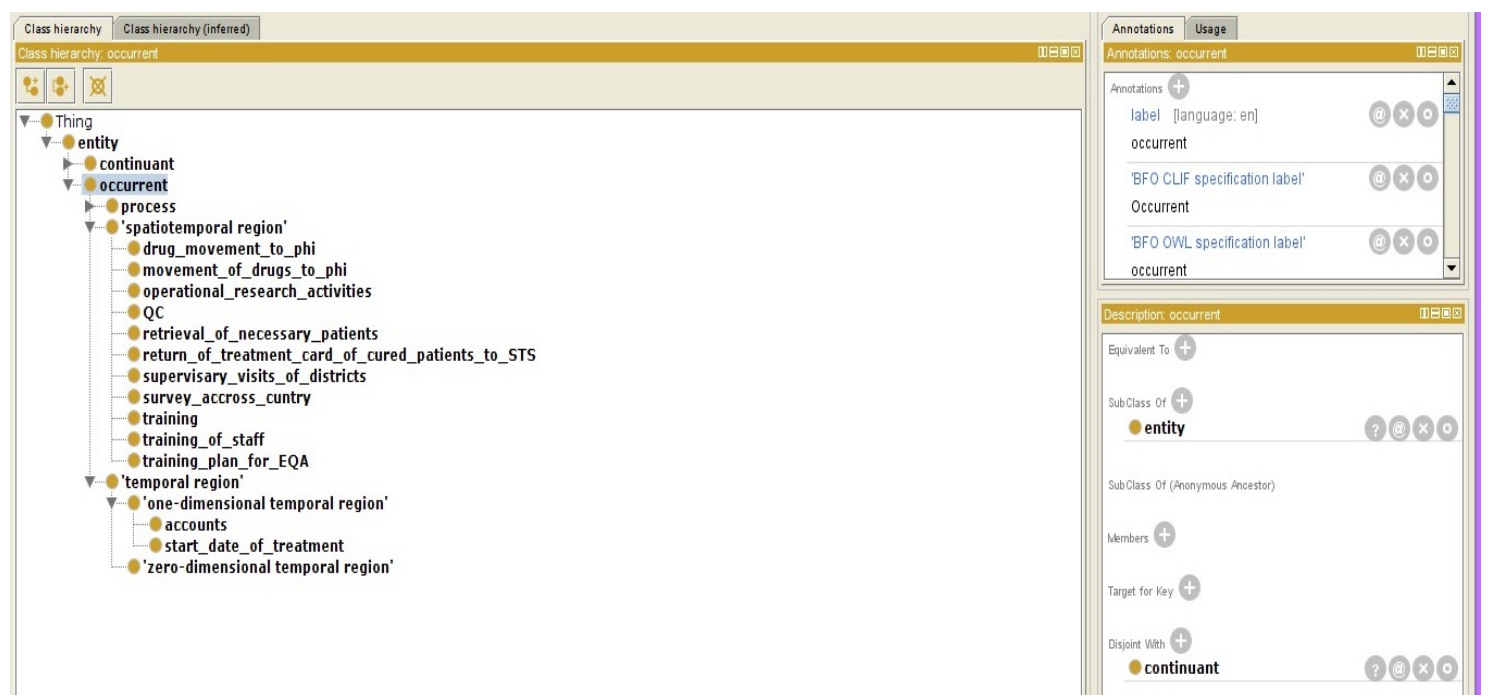

Fig. 10 Entities as Spatiotemporal Region and Temporal Region

\section{Diagnosis AND Treatment}

Tuberculosis is a disease which will infect or infest a human being. Considering human beings (patients) to be the centre of importance in our domain (decision support system) they are realized as an 'object'. Now this defines tuberculosis to be a disease, whose existence and disappearance will affect the actual object i.e. the patient. Hence Tuberculosis can be realized as a 'disposition'.

Disease is essentially a disposition which an organism bears because of one or more disorders present in its biological system. Considering diseases to be dispositions explains the fact that they can be present without any proper manifestation (i.e., without realization of the disposition) and that they can be realized in various kinds of manifestations (dependent for example on presence or absence of symptom-suppressant drugs). Resorting to dispositions, permits us to describe what an object can do and to have this description still be just even if relevant realizing processes never take place.

A proper study of signs and symptoms related to TB reveals that, considering as a whole, they are not instances of a single universal. In other words, sign and symptom are not natural kinds. Instead instances of each group consist of different types of entities comprising of material entities, processual entities, and qualities. Adherence to ontological realism, sign and symptom cannot be realized as named universal classes in an ontology. The propose work makes use of relations (properties) to connect entities that can be a sign, symptom, or both to the diseases, to which they relate.

The symptoms are being considered to be an occurrent as it tends to unfolds itself. It has subclasses like fever, cough, etc. An instance of the class patient will bear one or more of these symptoms which are implemented by use of corresponding data properties.

The suspected patients for TB may fall in any one of the categories namely (i) Pulmonary TB, (ii) Extrapulmonary TB,(iii) Pediatric TB (iii) Multi-Drug Resistance TB(MDR)/Extensively Drug resistant TB and (iv)TB/HIV

The above categories follow separate treatment regime and diagnosis regime developed by RNTCP, which based completely on WHO Stop TB strategy.

The proposed system uses rules extensively to direct the handler to take necessary steps as per the situation of the patient. The knowledge base that has been created cannot infer new knowledge. Rules act upon the knowledge base to extract the required knowledge.

The system presented in the paper focuses on care and management of patient for the following categories of the TB namely:

(i) Pulmonary TB(PTB)

(ii) Pediatric TB

(iii) Extra-Pulmonary TB(EPTB)

The RNTCP diagnostic algorithm for detection and treatment of the patients of the above mentioned categories were implemented in form of rules using SWRL(Semantic Web Rule Language), based on OWLDL(Description Logic) and Horn Logic. The rules were first written in SWRL and then implemented using PELLET reasoner. 
Table I indicates the RNTCP guidelines for detection and patient management of patient with suspected symptoms of extra-pulmonary TB, represented using SWRL

TABLE I. EXTRA PULMONARY TB DiAgNOSTIC ALGORITHM

\begin{tabular}{|c|c|}
\hline Label & $\begin{array}{l}\text { SWRL rules for extra-pulmonary TB patients } \\
\text { ( Based on Description Logic) }\end{array}$ \\
\hline E1 & $\begin{array}{l}\text { patient(?x) } \wedge \text { hasLymphEnlargementValue(?x, ?v) } \wedge \text { swrlb:greaterThan(?v, 2) -> } \\
\text { isPrescribedAntibiotics(?x, true) }\end{array}$ \\
\hline E2 & patient(?x) $\wedge$ hasAbscess(?x, true) -> isPrescribedAntibiotics(?x, true) \\
\hline E3 & $\begin{array}{l}\text { patient }(? \mathrm{x}) \wedge \text { hasLymphNodeEnlargement(?x, true) } \wedge \text { isPrescribedAntibiotics(?x, true) }-> \\
\text { undergoes(?x, FNAC) } \wedge \text { undergoes(?x, Smear_ZN_Stain_test) } \wedge \text { hasTBLymphadenitis(?x, } \\
\text { true) }\end{array}$ \\
\hline E4 & $\begin{array}{l}\text { patient(?x) ^ undergoes(?x, FNAC) } \wedge \text { hasResultOfFnac(?x, ?v) } \wedge \text { swrlb:equal(?v, } \\
\text { "positive") -> prescribedcategory3Treatment(?x, true) }\end{array}$ \\
\hline E5 & $\begin{array}{l}\text { patient(?p) } \wedge \text { undergoes(?p, FNAC) } \wedge \text { hasResultOfFnac(?p, ?value) } \wedge \text { swrlb:equal(?value, } \\
\text { "negative") -> undergoes(?p, Excision_Biopsy) } \wedge \text { undergoes(?p, Histo- } \\
\text { pathological_examination) }\end{array}$ \\
\hline E6 & $\begin{array}{l}\text { patient(?p) } \wedge \text { undergoes(?p, Excision_Biopsy) } \wedge \text { hasResultOfExcisionBiopsy(?p, ?value) } \wedge \\
\text { swrlb:equal(?value, "positive") -> prescribedcategory3Treatment(?p, true) }\end{array}$ \\
\hline E7 & $\begin{array}{l}\text { patient(?p) } \wedge \text { undergoes(?p, Histo-pathological_examination) } \wedge \\
\text { hasResultOfHistoPathologicalExamination(?p, ?value) } \wedge \text { swrlb:equal(?value, "positive") - } \\
>\text { prescribedcategory3Treatment(?p, true) }\end{array}$ \\
\hline
\end{tabular}

Fig.11 depicts the implementation of extra-pulmonary TB diagnostic algorithm, patient with suspected symptom's are entered as data type properties, which acts as antecedant (body) of the rule.

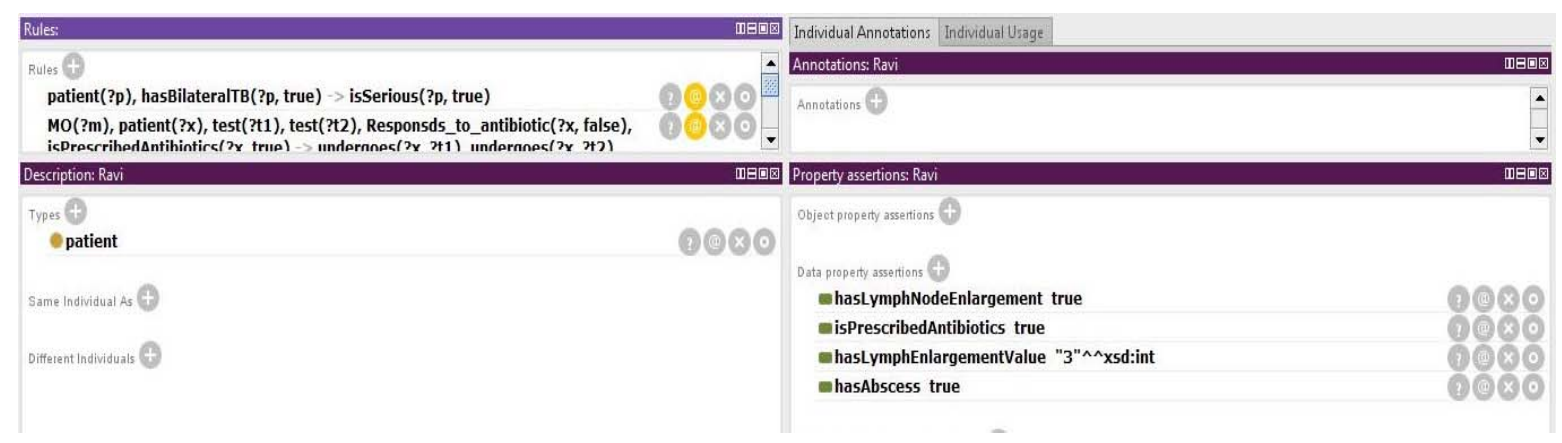

Fig. 11 Patient with suspected symptoms of EPTB

The data properties in Fig. 11 acts as input for reasoner, once reasoned is started, consequent part (head) of the rule is inferred as depicted in Fig. 12.

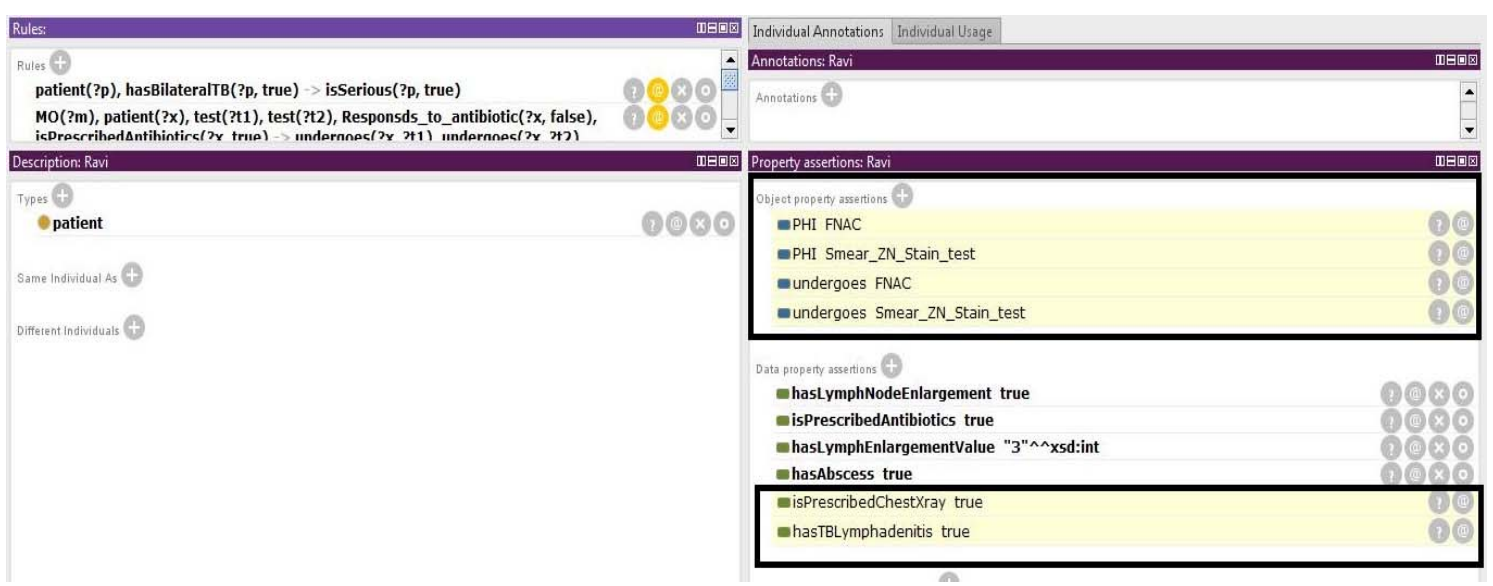

Fig. 12 Outcomes inferred by reasoned for patients with EPTB symptoms 
Fig. 12 indicates the results inferred by the reasoner (rectangular box) which states the outcome derived and necessary action/test to be performed. Fig. 13 depicts the category of treatment that has been inferred to be given to the patient with suspected symptom of extra-pulmonary TB after getting the prescribed test result.

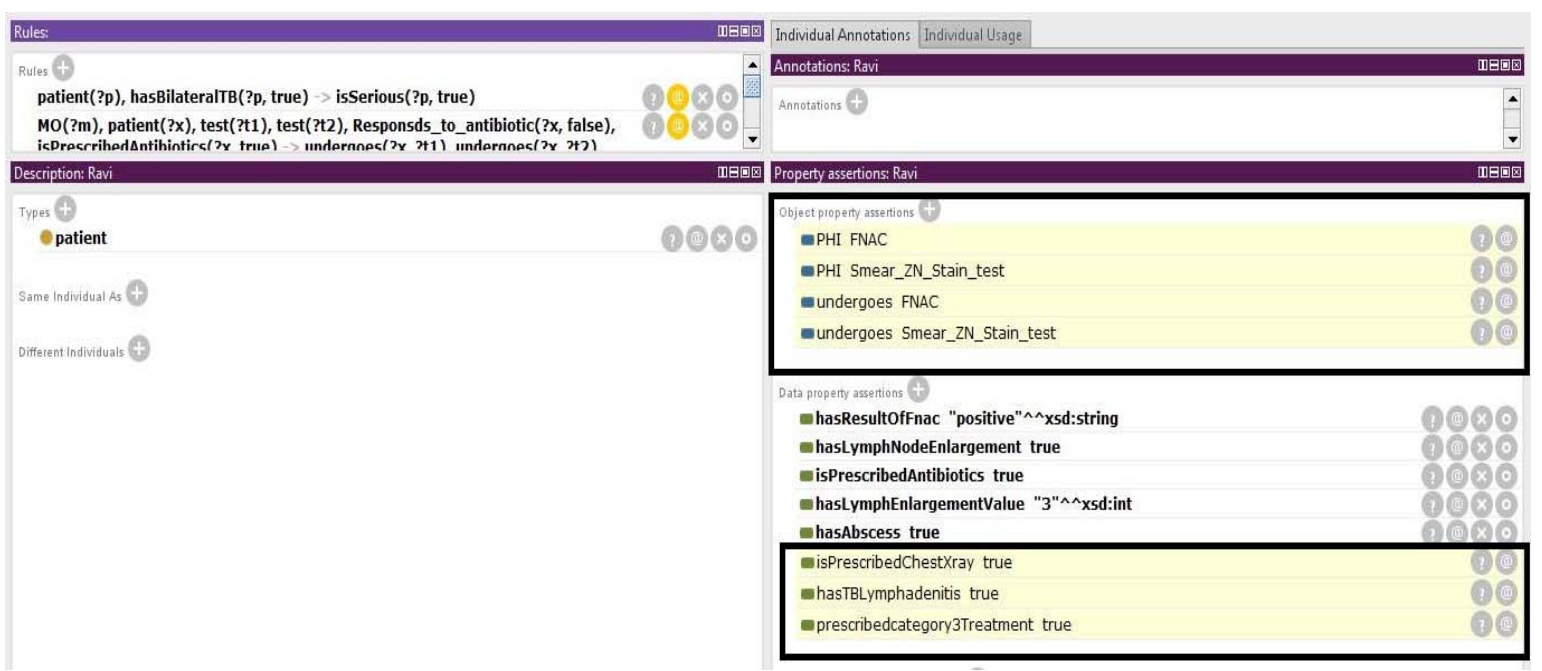

Fig. 13 Outcome inferred by the reasoned after prescribed EPTB test

Similarly the diagnostic algorithm for pulmonary TB patients was implemented in similar manner. An instance under patient was created and susceptive symptoms were added as data properties and then reasoned were used for determining the necessary action/outcome.

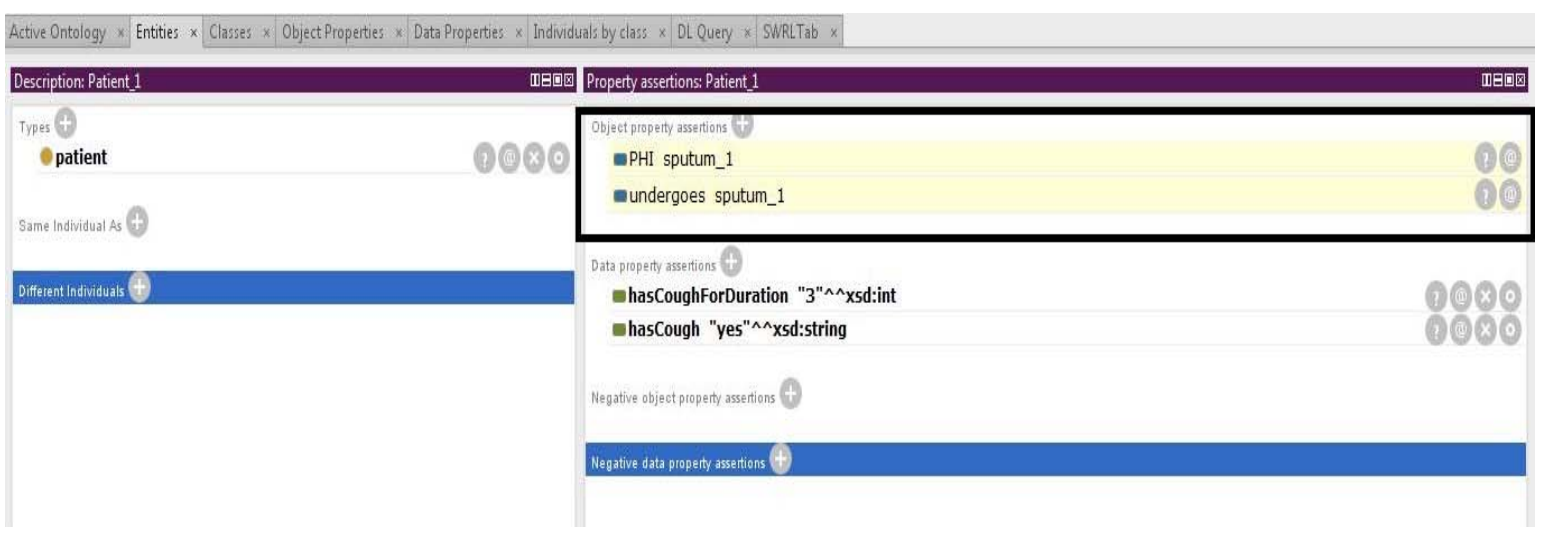

Fig. 14 Patient with suspected symptoms of PTB

Fig. 14 indicates the inferred action (rectangular box) based on the symptom reported by the patients. Fig. 15 indicates the outcome after patient undergoes first sputum smear test result.

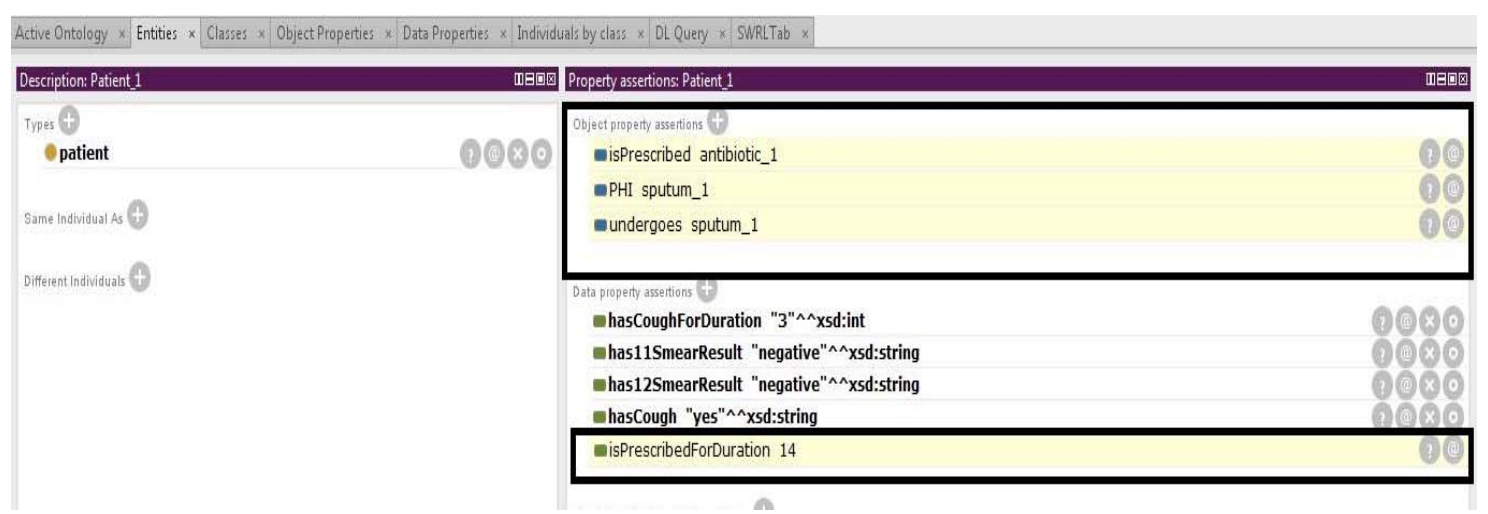

Fig. 15 Outcome after first negative Sputum smear test

Fig. 16 indicates the outcomes after patient undergoes second sputum smear test after having antibiotics and still having TB suspected symptoms. Fig. 17 indicates outcome as patient is being categorized and sputum negative PTB and treatment is started. 


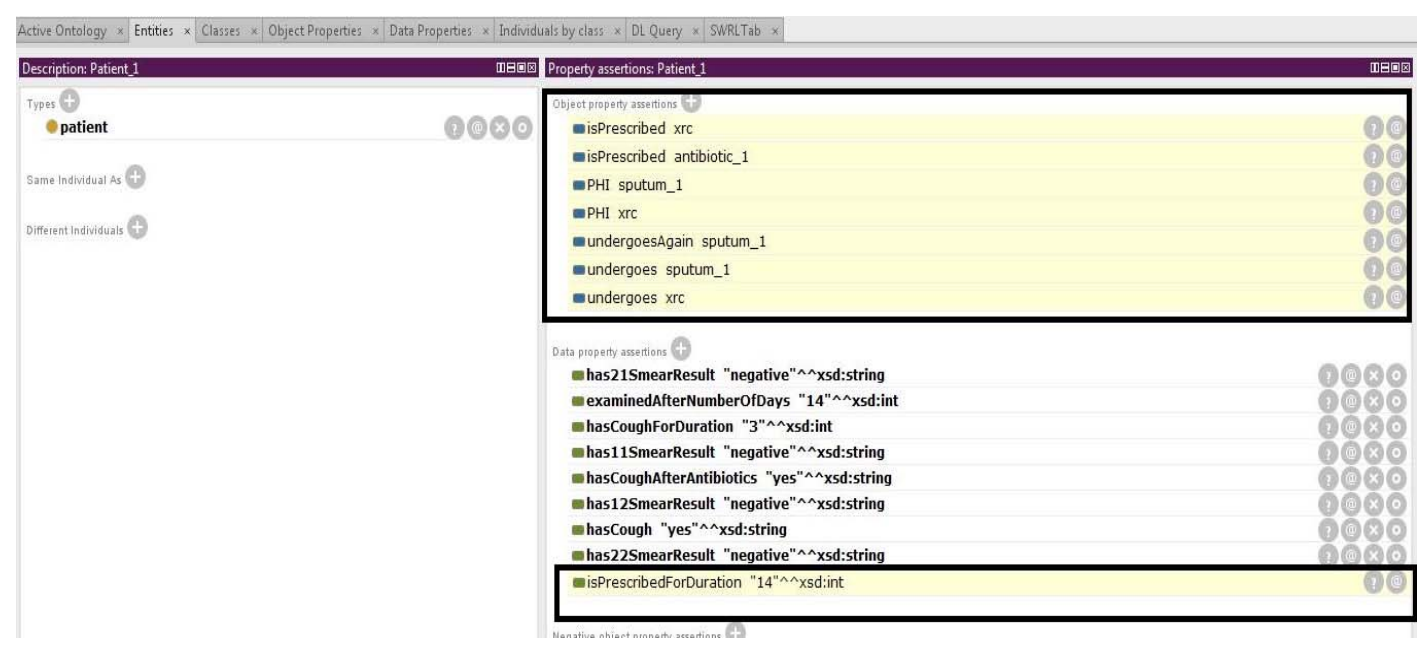

Fig. 16 Outcome after second negative Sputum smear test

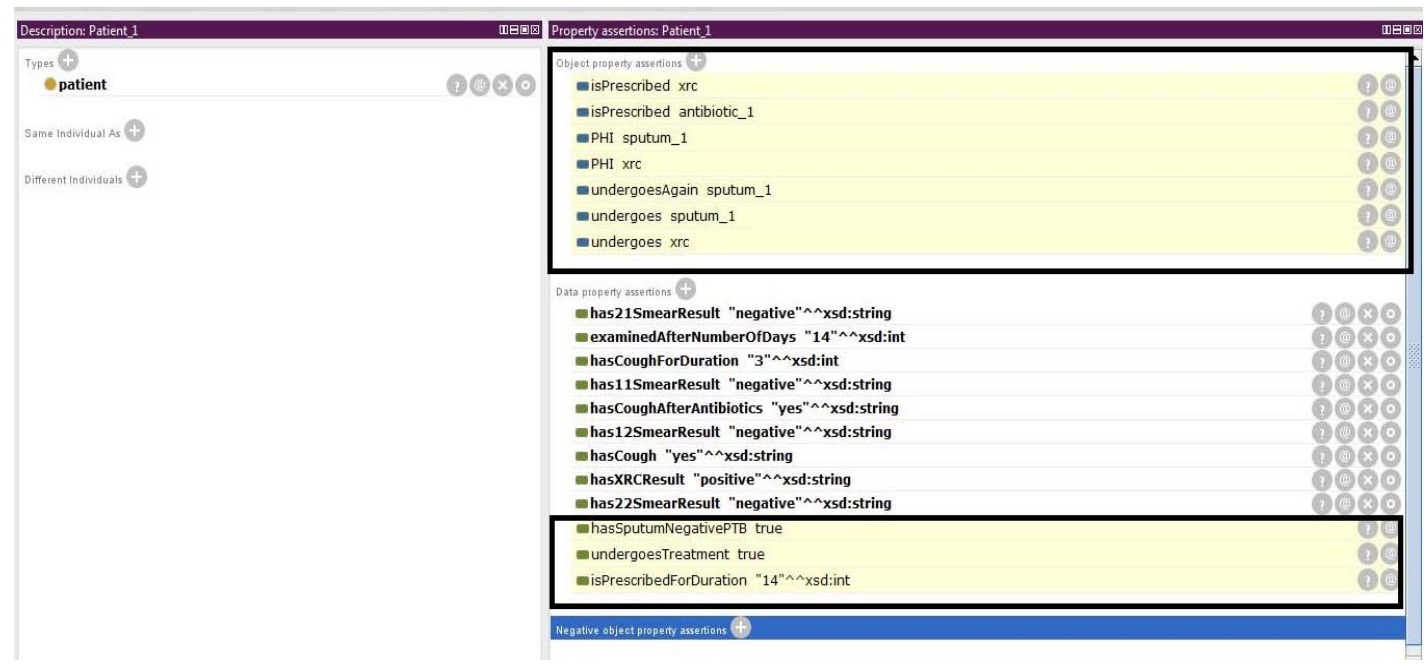

Fig. 17 Outcome after X-ray for Chest

Fig. 18 indicates the inferred outcome for a patient categorized as Sputum positive TB based on sputum smear test, it also provides the details of category and treatment regime.

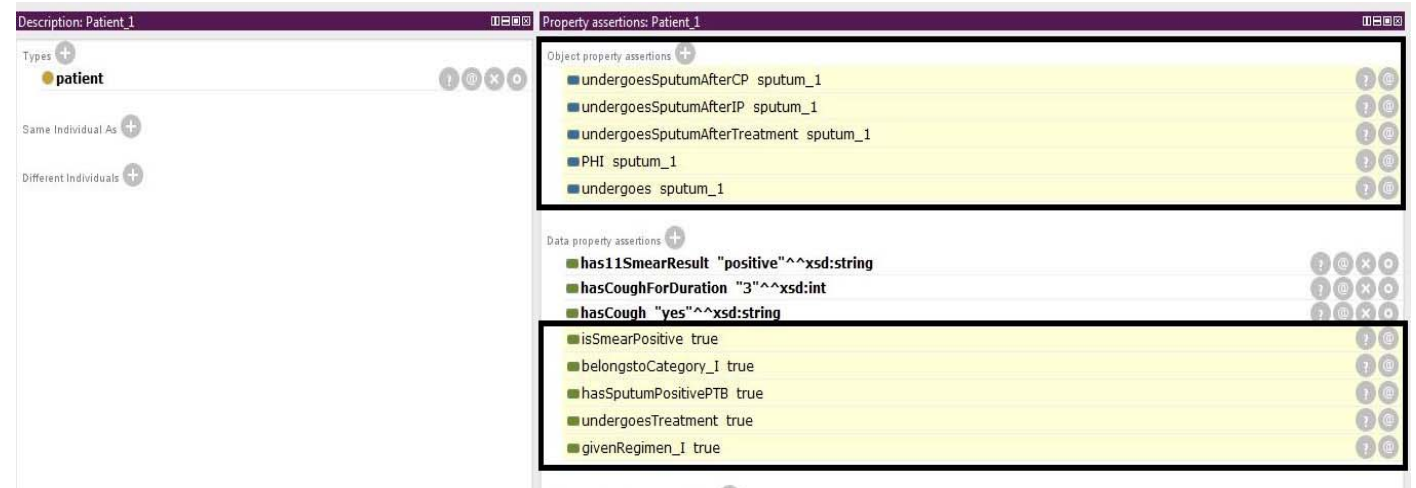

Fig. 18 Patient diagnosed with Smear Positive TB

Table II indicates the RNTCP guidelines for detection and patient management of patient with suspected symptoms of pulmonary TB , represented using SWRL 
TABLE II. PUlmonary TB Diagnostic AlgorithM

\begin{tabular}{|l|l|}
\hline S.No. & \multicolumn{1}{c|}{$\begin{array}{c}\text { SWRL rules for Pulmonary TB patients } \\
\text { ( Based on Description Logic) }\end{array}$} \\
\hline 1 & $\begin{array}{l}\text { patient(?x) } \wedge \text { hasCough(?x, ?value) } \wedge \text { hasCoughForDuration(?x, ?week) } \wedge \\
\text { swrlb:equal(?value, "yes") } \wedge \text { swrlb:greaterThan(?week, 2) -> undergoes(?x, sputum_1) }\end{array}$ \\
\hline 2 & $\begin{array}{l}\text { antibiotics(?a) } \wedge \text { patient(?x) } \text { ( has11SmearResult(?x, ?v) } \wedge \text { has12SmearResult(?x, ?v1) } \wedge \\
\text { swrlb:equal(?v, "negative") } \wedge \text { swrlb:equal(?v1, "negative") -> isPrescribed(?x, ?a) } \wedge\end{array}$ \\
\hline 3 & $\begin{array}{l}\text { undergoesAgain(?x, ?s) } \wedge \text { has21SmearResult(?x, ?v) } \wedge \text { has22SmearResult(?x, ?v1) } \wedge \\
\text { swrlb:equal(?v, "negative") } \wedge \text { swrlb:equal(?v1, "negative") -> isPrescribed(?x, xrc) } \wedge\end{array}$ \\
\hline 4 & $\begin{array}{l}\text { patient(?x) } \wedge \text { has11SmearResult(?x, ?v1) } \wedge \text { swrlb:equal(?v1, "positive") -> } \\
\text { hasSputumPositivePTB(?x, true) }\end{array}$ \\
\hline 5 & $\begin{array}{l}\text { patient(?p) } \wedge \text { isSmearPositive(?p, true) -> belongstoCategory_I(?p, true) } \\
\text { patient(?x) } \wedge \text { belongstoCategory_I(?x, true) -> givenRegimen_I(?x, true) }\end{array}$ \\
\hline 7 & $\begin{array}{l}\text { patient(?p) } \wedge \text { completedTreatment(?p, true) } \wedge \text { isCured(?p, true) } \wedge \\
\text { reportsBackWithSymptom(?p, true) -> undergoesAgain(?p, sputum_1) }\end{array}$ \\
\hline 8 & $\begin{array}{l}\text { patient(?p) } \wedge \text { undergoesAgain(?p, sputum_1) } \wedge \text { has31SmearResult(?p, ?value) } \wedge \\
\text { swrlb:equal(?value, "positive") -> isRelapse(?p, true) }\end{array}$ \\
\hline 9 & $\begin{array}{l}\text { patient(?p) } \wedge \text { undergoesAgain(?p, sputum_1) } \wedge \text { has32SmearResult(?p, ?value) } \wedge \\
\text { swrlb:equal(?value, "positive") -> isRelapse(?p, true) }\end{array}$ \\
\hline
\end{tabular}

The implementation of diagnostic algorithm for paediatric pulmonary tuberculosis was done in similar manner as discussed above. Fig. 19 depicts an inferred outcome (rectangular box) for an instance Chotu of patient which has reported with suspected symptoms of paediatric pulmonary TB and has undergone sputum smear test. The category of patient are also mentioned in outcome.

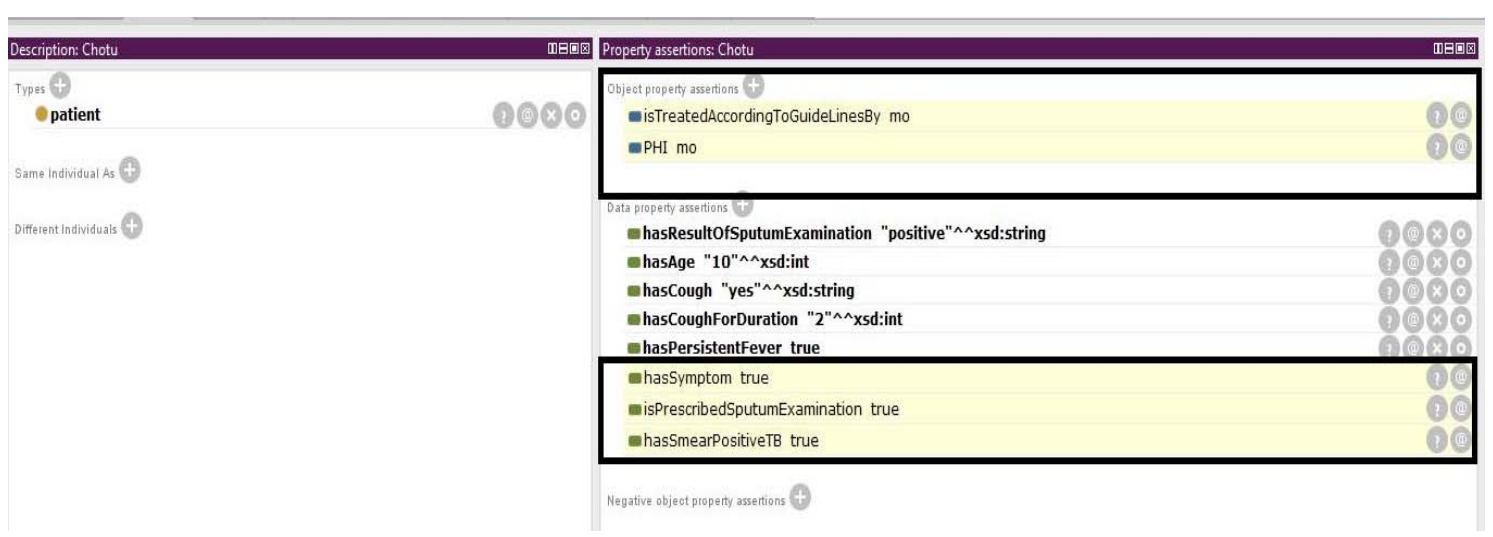

Fig. 19 Patient with Paediatric Pulmonary TB symptoms

Fig. 20 indicates the inferred outcome of patient Chotu with suspected symptoms and negative result of sputum smear examination. 


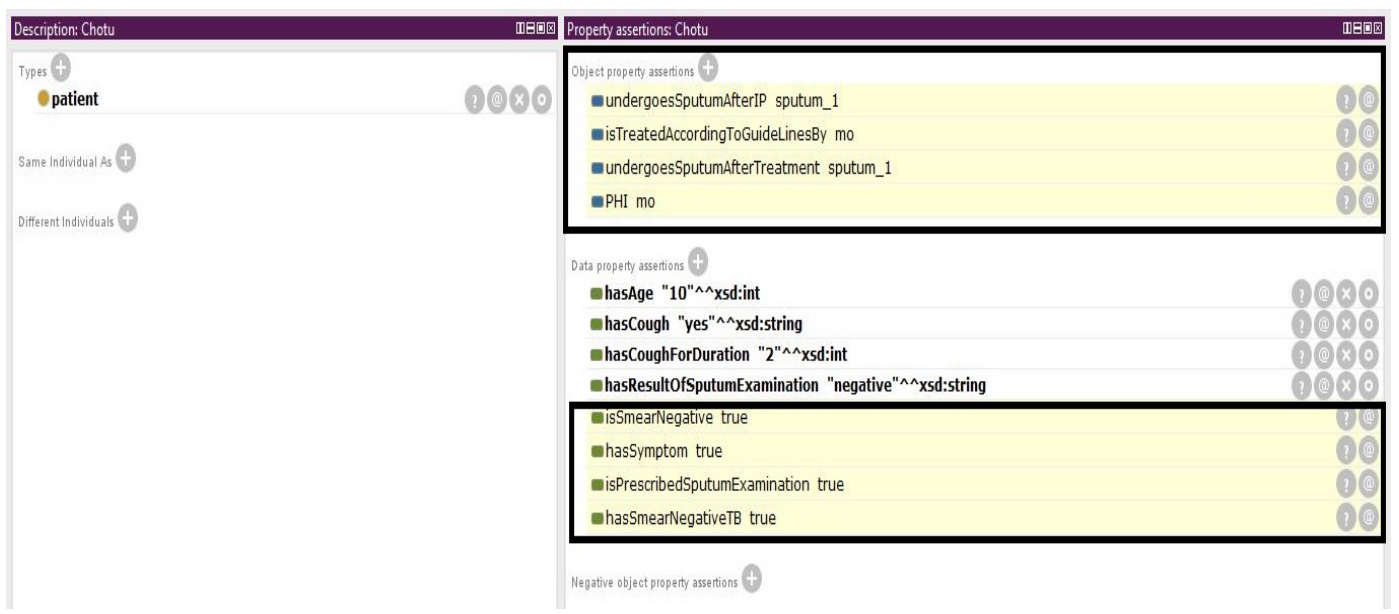

Fig. 20 Patient categorized as smear negative

Fig. 21 depicts the inferred actions to be taken for patient with smear negative pulmonary pediatrics TB who reports back with symptoms even after having prescribed course of antibiotics

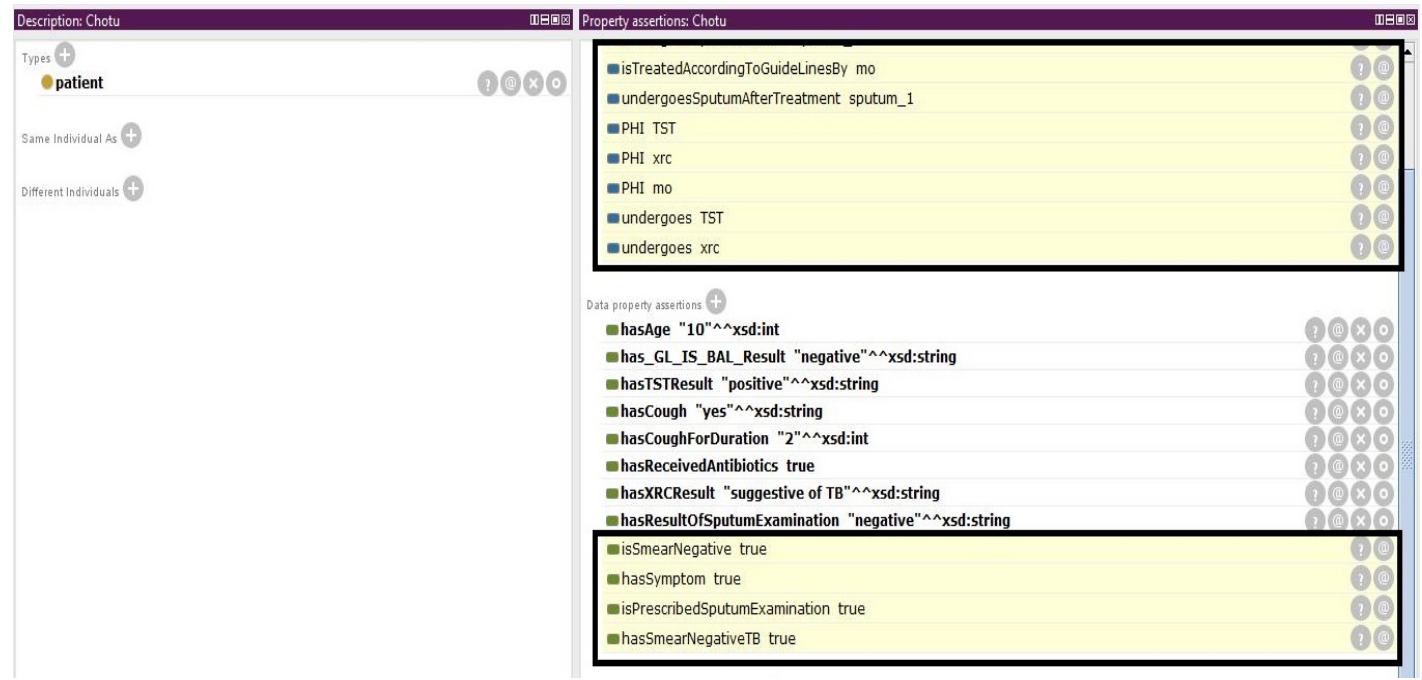

Fig. 21 Patient with symptoms after prescribed antibiotics

Table III indicates the RNTCP guidelines for detection and patient management of patient with suspected symptoms of Paediatric pulmonary TB, represented using SWRL

TABLE III. Paediatric Pulmonary TB Diagnostic Algorithm

\begin{tabular}{|l|l|}
\hline S.No. & \multicolumn{1}{|c|}{$\begin{array}{l}\text { SWRL rules for Paediatric pulmonary TB patients } \\
\text { ( Based on Description Logic) }\end{array}$} \\
\hline 1 & $\begin{array}{l}\text { patient(?x) } \wedge \text { hasCough(?x, ?v) } \wedge \text { swrlb:equal(?v, "yes") } \wedge \text { hasCoughForDuration(?x, ?v1) } \wedge \\
\text { swrlb:equal(?v1, 2) -> hasSymptom(?x, true) }\end{array}$ \\
\hline 2 & patient(?x) $\wedge$ hasPersistentFever(?x, ?v2) $\wedge$ swrlb:equal(?v2, true) -> hasSymptom(?x, true) \\
\hline 3 & patient(?p) $\wedge$ hasLossOfWeight(?p, true) -> hasSymptom(?p, true) \\
\hline 4 & patient(?p) $\wedge$ hasHistoryOfContact(?p, true) -> hasSymptom(?p, true) \\
\hline 5 & $\begin{array}{l}\text { patient(?x) } \wedge \text { hasAge(?x, ?V) } \wedge \text { swrlb:lessThanOrEqual(? } \text {, 14) } \wedge \text { hasSymptom(?x, true) }-> \\
\text { isPrescribedSputumExamination(?x, true) }\end{array}$ \\
\hline 6 & $\begin{array}{l}\text { patient(?x) } \wedge \text { hasResultOfSputumExamination(?x, ?value) } \wedge \\
\text { isPrescribedSputumExamination(?x, true) } \wedge \text { swrlb:equal(?value, "positive") -> } \\
\text { hasSmearPositiveTB(?x, true) }\end{array}$ \\
\hline 7 & $\begin{array}{l}\text { patient(?x) } \wedge \text { hasResultOfSputumExamination(?x, ?value) } \wedge \\
\text { isPrescribedSputumExamination(?x, true) } \wedge \text { swrlb:equal(?value, "negative") -> } \\
\text { isSmearNegative(?x, true) }\end{array}$ \\
\hline
\end{tabular}




\begin{tabular}{|c|c|}
\hline 8 & $\begin{array}{l}\text { patient(?p) } \wedge \text { isSmearNegative(?p, true) } \wedge \text { hasReceivedAntibiotics(?p, true) -> } \\
\text { isPrescribed(?p, xrc) } \wedge \text { isPrescribed(?p, TST) }\end{array}$ \\
\hline 9 & $\begin{array}{l}\text { patient(?x) } \wedge \text { hasXRCResult(?x, ?v1) } \wedge \text { swrlb:equal(?v1, "suggestive of TB") } \\
\text { hasTSTResult(?x, ?v2) } \wedge \text { swrlb:equal(?v2, "positive") -> isPrescribed(?x, GL_IS_BAL) }\end{array}$ \\
\hline 10 & $\begin{array}{l}\text { patient(?p) } \wedge \text { has_GL_IS_BAL_Result(?p, ?x) } \wedge \text { swrlb:equal(?x, "positive") -> } \\
\text { isSmearPositive(?p, true) } \wedge \text { hasSmearPositiveTB(?p, true) }\end{array}$ \\
\hline 11 & $\begin{array}{l}\text { patient(?p) } \wedge \text { has_GL_IS_BAL_Result(?p, ?x) } \wedge \text { swrlb:equal(?x, "negative") -> } \\
\text { hasSmearNegativeTB(?p, true) }\end{array}$ \\
\hline 12 & $\begin{array}{l}\text { patient(?p) } \wedge \text { hasXRCResult(?p, ?x) } \wedge \text { swrlb:equal(?x, "normal") } \wedge \text { hasTSTResult(?p, ?x1) } \wedge \\
\text { swrlb:equal(?x1, "negative") -> isReviewedForAlternateDiagnosis(?p, true) }\end{array}$ \\
\hline 13 & $\begin{array}{l}\text { patient(?p) } \wedge \text { isReviewedForAlternateDiagnosis(?p, true) } \wedge \mathrm{MO}(? \mathrm{~m}) \wedge \\
\text { hasEstablishedAlternative_Diagnosis(?m, true) -> isPrescribed(?p, Specific_Therapy) }\end{array}$ \\
\hline
\end{tabular}

The proposed system also keeps track of various statistic related to the patient for example number of people cured or died etc.

SQWRL(Semantic Query-enhanced Web Rule Language Semantic Query-enhanced Web Rule Language)is used by the proposed system to accomplish these above tasks.

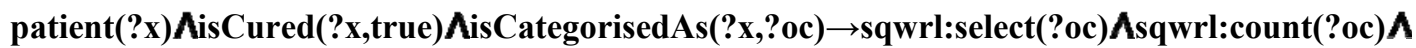 sqwrl:columnNames("case", "count")}

The above query will count the number of patients that have been cured and the system will store the result as shown in Fig. 22.

\begin{tabular}{|c|c|c|c|c|}
\hline \multicolumn{5}{|c|}{ Active Ontology $\times$ Entities $\times$ Classes $\times$ Object Properties $\times$ Data Properties $\times$ Individuals by class $\times$ DL Query $\times$ SWRLTab $\times$ SQWRLTab $\times$} \\
\hline & & Name & Rule & Comment \\
\hline$\nabla$ & nd3 & & patient(?p) DinNonRNTCPregion(Pp, true) D hasextraPulmonaryTB(?p, true) - belongstoND1(?p, true) & \\
\hline$\checkmark$ & nd4 & & patient??p) D inNonRNTCPregion(?p, true) D isSmearNegative(?p, true) DisSerious(?p, false) - belongstoND2(?p, true) & \\
\hline$\checkmark$ & nd5 & & patient(?p) DinNonRNTCPregion(?p, true) D hasextraPulmonaryTB(?p, true) DisSerious(?p, false) - belongstoND2(?p, true) & \\
\hline$\checkmark$ & 99 & & & \\
\hline [ & lag9 & & 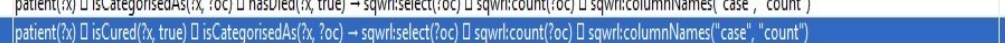 & \\
\hline$\checkmark$ & r1 & & patient(?x) प hasSymptom(?x, true) - isPrescribedSputumExamination(?x, true) & \\
\hline$\checkmark$ & 12 & & 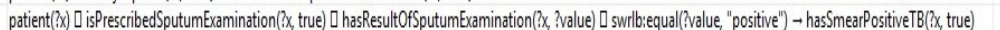 & \\
\hline$\checkmark$ & 13 & & 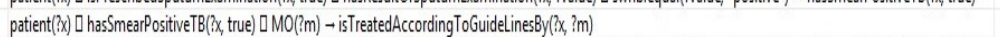 & \\
\hline$\checkmark$ & 14 & & 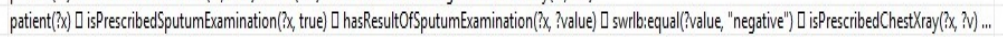 & \\
\hline$\checkmark$ & 15 & & 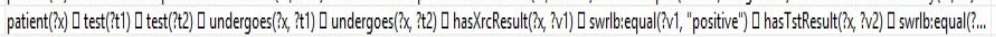 & \\
\hline$\checkmark$ & 16 & & patient(?x) Dhastest3Result(?', ?value) D swrlb:equal('value, "positive") - hasSmearPositiveTB("X, true) & \\
\hline$\checkmark$ & 17 & & patient(?x) [ hastest3Result([x, ?value) D swrlb:equal(?value, "negative") - hasSmearNegativeTB(?x, true) & \\
\hline$\checkmark$ & & & patient(?x) DhasSmearNegativeTB(?x, true) DMO(?m) $\rightarrow$ is TreatedAccordingToGuidelinesBy(?x, ?m) & \\
\hline \multirow[t]{2}{*}{ 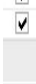 } & & & 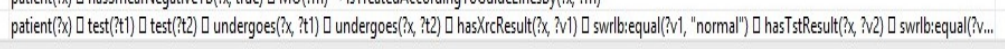 & \\
\hline & & & & New Edit Delet \\
\hline \multicolumn{5}{|c|}{ SQWRL Queries OWL 2RL 99999} \\
\hline \multicolumn{4}{|r|}{ case } & count \\
\hline cure & & & 2 & \\
\hline
\end{tabular}

Fig. 22 Result of SQWRL query

\section{CONCLUSION AND FUtURE SCOPE}

The Ontology developed is capable of representing an entire knowledge base in form of Ontology-Graphs. This knowledge base includes organizational structure of the Nationwide Programme. The inclusion of SWRL for implementing the diagnosis and treatment process is the actual heart and soul of the Decision Support System. Various actors involved at different levels are the most likely target audience to draw benefit in terms of decision making. Expanding the use SWRL, the proposed system will also enable the medical practitioners to keep count of outcome of treatment namely, number of patients cured, defaulted, died, etc. SPARQL enables us to retrieve functions of individual actors involved at various levels. Major difficulty which arose was the incompatibility issues between different versions of the Protégé. Ontologies are now being used in Information Science as an efficient tool for performing a set of tasks like enhancing communication between user and machine. It also utilises any existing data model or knowledge schema. As a result, basic concept of semantic web has matured to a higher level and different kinds of ontologies have been designed. Amongst different categories and classifications, BFO is the only one which supports reasoning along with the general Open World Semantics followed by all Ontologies. The proposed Decision Support System makes uses of the Knowledge Base in combination with SWRL to get a more desirable and logic based solution considering the domain of treatment for Tuberculosis 
The proposed ontology can be extended for of MDR-TB (Multi Drug Resistance) and HIV-TB positive category of patients and their respective treatment regimen. The proposed Decision Support System can be enhanced to track the individual patients in case of relocation. Further, a web integration of the entire Decision Support System is yet to be implemented for the sake of the end users. However, from the developer's point of view ontology can be used to deduce implicit knowledge and arrive at a particular decision if the case so demands.

\section{REFERENCES:}

[1] Berners-Lee, Tim, James Hendler, and Ora Lassila. "The semantic web." Scientific american 284.5 (2001): 28 -37.

[2] Shadbolt, Nigel, Wendy Hall, and Tim Berners-Lee. "The semantic web revisited." Intelligent Systems, IEEE 21.3 (2006): $96-101$.

[3] McGuinness, Deborah L., and Frank Van Harmelen. "OWL web ontology language overview." W3C recommendation 10, no. 10 (2004): 2004

[4] McIlraith, S. A., Son, T. C., \& Zeng, H. (2001). Semantic web services. IEEE intelligent systems, (2), 46-53.

[5] Blomqvist, E. (2014). The use of Semantic Web technologies for decision support-a survey. Semantic Web, 5(3), 177-201.

[6] Power, D. J. "Decision Support Systems: Concepts and Resources for Managers." Studies in Informatics and Control 11, no. 4 (2002): 349-350.

[7] Bray, Tim, Jean Paoli, C. Michael Sperberg-McQueen, Eve Maler, and François Yergeau. "Extensible markup language (XML)." World Wide Web Consortium Recommendation REC-xml-19980210. http://www. w3. org/TR/1998/REC-xml-19980210 16 (1998).

[8] Buranarach, Marut, Thepchai Supnithi, Noppadol Chalortham, Vasuthep Khunthong, Patcharee Varasai, and Asanee Kawtrakul. "A semantic web framework to support knowledge management in chronic disease healthcare." In Metadata and semantic research, pp. 164-170. Springer Berlin Heidelberg, 2009.

[9] RDF Semantic Web Standards https://www.w3.org/RDF/

[10] RDF Schema 1.1 http://www.w3.org/TR/rdf-schema/

[11] Rodríguez-González, Alejandro, Jose Emilio Labra-Gayo, Ricardo Colomo-Palacios, Miguel A. Mayer, Juan Miguel Gómez-Berbís, and Angel García-Crespo. "SeDeLo: using semantics and description logics to support aided clinical diagnosis." Journal of medical systems 36, no. 4 (2012): 2471-2481.

[12] Ceusters, Werner, and Barry Smith. "Referent tracking for treatment optimisation in schizophrenic patients: A case study in applying philosophical ontology to diagnostic algorithms." Web Semantics: Science, Services and Agents on the World Wide Web 4, no. 3 (2006): 229-236.

[13] Smith, Barry, and P. Grenon. "Basic formal ontology." Draft. Downloadable at http://ontology. buffalo. edu/bfo (2002).

[14] Abidi, Syed Sibte Raza. "Healthcare knowledge management: The art of the possible." In Knowledge management for health care procedures, pp. 1-20. Springer Berlin Heidelberg, 2008.

[15] Hussain, Sajjad, Samina Raza Abidi, and Syed Sibte Raza Abidi. "Semantic web framework for knowledge-centric clinical decision support systems." In Artificial intelligence in medicine, pp. 451-455. Springer Berlin Heidelberg, 2007.

[16] Bouamrane, Matt-Mouley, Alan Rector, and Martin Hurrell. "Using OWL ontologies for adaptive patient information modelling and preoperative clinical decision support." Knowledge and information systems 29, no. 2 (2011): 405-418.

[17] Wrighton, Naomi, and Christopher D. Buckingham. "Defining an OWL ontology that could be used to integrate mental-health risk information within a decision support system." In eHealth, Telemedicine, and Social Medicine, 2009. eTELEMED'09. International Conference on, pp. 245-250. IEEE, 2009.

[18] Turki, Mohamed, Gilles Kassel, Inès Saad, and Faïez Gargouri. "COOP: A core ontology of organization’s processes for group decision making." Journal of Decision Systems 23, no. 1 (2014): 55-68.

[19] Bottazzi, Emanuele, and Roberta Ferrario. "Preliminaries to a DOLCE ontology of organisations." International Journal of Business Process Integration and Management 4, no. 4 (2009): 225-238.

[20] Turcin, Ivan, Vladimir Ergovic, and Marko Lackovic. "Ontology Driven Decision Support System Architecture for Gait Analysis." In International Symposium on Biomedical Engineering and Medical Physics, 10-12 October, 2012, Riga, Latvia, pp. 78-81. Springer Berlin Heidelberg, 2013.

[21] Chai, Junyi, and James NK Liu. "An ontology-driven framework for supporting complex decision process." In World Automation Congress (WAC), 2010, pp. 1-6. IEEE, 2010.

[22] Ishizu, Syohei, Andreas Gehrmann, Junya Minegishi, and Yoshimitsu Nagai. "Ontology-driven decision support systems for management system audit." In Proceedings of the 52nd Annual Meeting of the ISSS-2008, Madison, Wisconsin, vol. 3, no. 1. 2008.

[23] Wang, Hsien-Tseng and Tansel, Abdullah Uz (2013) "Composite Ontology-Based Medical Diagnosis Decision Support System Framework," Communications of the IIMA: Vol. 13: Iss. 2, Article 4.

[24] Ding, Chaohai, Mike Wald, and Gary Wills. "Linked data-driven decision support for accessible travelling." In Proceedings of the 12th Web for All Conference, p. 39. ACM, 2015.

[25] Saremi, Amin, Mostafa Esmaeili, Jaafar Habibi, and Arman Ghaffari. "O2dss: A framework for ontology-based decision support systems in pervasive computing environment." In Modeling \& Simulation, 2008. AICMS 08. Second Asia International Conference on, pp. 41-45. IEEE, 2008.

[26] Ceccaroni, Luigi, Ulises Cortés, and Miquel Sanchez-Marre. "OntoWEDSS: augmenting environmental decision-support systems with ontologies." Environmental Modelling \& Software 19, no. 9 (2004): 785-797.

[27] Ceccaroni, Luigi, Ulises Cortés, and Miquel Sànchez-Marrè. "WaWO-An ontology embedded into an environmental decision-support system for wastewater treatment plant management." (2000).

[28] Rospocher, Marco, and Luciano Serafini. "Ontology-centric Decision Support." In SeRSy, pp. 61-72. 2012

[29] Rospocher, M. Pescado ontology documentation. Tech. Rep., 2010.[Online]. Available: http://www. pescado-project. eu/Pages/Pdfspages/PESCaDO Ontology Documentation 2.0. pdf.

[30] Happel, Hans-Jörg, and Stefan Seedorf. "Applications of ontologies in software engineering." In Proc. of Workshop on Sematic Web Enabled Software Engineering"(SWESE) on the ISWC, pp. 5-9. 2006.

[31] Oberle, Daniel. "How ontologies benefit enterprise applications." Semantic Web Journal-Interoperability, Usability, Applicability (2009).

[32] Carvalho, Rommel Novaes. "Representation and Modeling Methodology." PhD diss., George Mason University, 2011.

[33] Carvalho, Rommel N., Kathryn B. Laskey, Paulo Cesar G. da Costa, Marcelo Ladeira, Laécio L. Santos, and Shou Matsumoto. "Probabilistic Ontology and Knowledge Fusion for Procurement Fraud Detection in Brazil." URSW 527 (2009): 3-14.

[34] Laskey, Kathryn B., Richard Haberlin, Rommel N. Carvalho, and Paulo Cesar G. da Costa. "PR-OWL 2 Case Study: A Maritime Domain Probabilistic Ontology." In STIDS, pp. 76-83. 2011.

[35] Peters, Bjoern, and OBI Consortium. "Ontology for biomedical investigations." (2009). 
[36] Spear, Andrew D. "Ontology for the twenty first century: An introduction with recommendations." Institute for Formal Ontology and Medical Information Science, Saarbrücken, Germany (2006).

[37] Smith, B., Almeida, M., Bona, J., Brochhausen, M., Ceusters, W., Courtot, M., ... \& Hogan, W. (2012). Basic Formal Ontology 2.0 Draft Specification and User's Guide.

[38] Arp, R., Smith, B., \& Spear, A. D. (2015). Building ontologies with basic formal ontology. Mit Press.

[39] Technical and Operational Guidelines for Tuberculosis Control, Central TB Division Directorate General of Health Services Ministry of Health and Family Welfare, Government of India (2003).

[40] O'Connor, M., \& Das, A. (2009, October). SQWRL: a query language for OWL. In Proceedings of the 6th International Conference on OWL: Experiences and Directions-Volume 529 (pp. 208-215). CEUR-WS. org.

[41] Horrocks, I., Patel-Schneider, P. F., Boley, H., Tabet, S., Grosof, B., \& Dean, M. (2004). SWRL: A semantic web rule language combining OWL and RuleML. W3C Member submission, 21, 79.

[42] Zaniolo, C., \& Saccá, D. (1987, May). Implementation of recursive queries for a data language based on pure horn logic. In Proceedings of the 4th International Conference on Logic Programming (Melbourne (pp. 104-135).

[43] Grosof, B. N., Horrocks, I., Volz, R., \& Decker, S. (2003, May). Description logic programs: combining logic programs with description logic. In Proceedings of the 12th international conference on World Wide Web (pp. 48-57). ACM.

[44] Parsia, B., \& Sirin, E. (2004, November). Pellet: An owl dl reasoner. In Third International Semantic Web Conference-Poster (Vol. 18)

[45] Sheth, A., Henson, C., \& Sahoo, S. S. (2008). Semantic sensor web. IEEE Internet computing, 12(4), 78-83.

[46] Sheth, A., Anantharam, P., \& Henson, C. (2013). Physical-cyber-social computing: An early 21st century approach. IEEE Intelligent Systems, 28(1), 78-82.

[47] Sahoo, S. S., Weatherly, D. B., Mutharaju, R., Anantharam, P., Sheth, A., \& Tarleton, R. L. (2009, November). Ontology-driven provenance management in escience: An application in parasite research. In OTM Confederated International Conferences" On the Move to Meaningful Internet Systems" (pp. 992-1009). Springer Berlin Heidelberg.

[48] Sheth, A., Anantharam, P., \& Thirunarayan, K. (2014). kHealth: Proactive Personalized Actionable Information for Better Healthcare. In Workshop on Personal Data Analytics in the Internet of Things (PDA@ IOT 2014), collocated at VLDB.

\section{AUTHOR PROFILE}
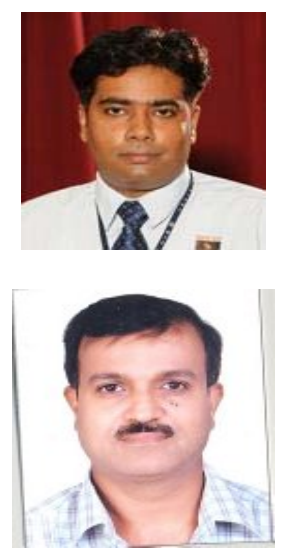

Kumar Abhishek is working as an Assistant Professor, Dept. of Computer Science and Engineering, National Institute of Technology, Patna, Bihar, India. His area of interest lies in RDF also in Semantic Web, Ontology, Semantic Sensor Web and Big data.

Maheshwari Prasad Singh (M.P.Singh) is working as an Assistant Professor, Dept. of Computer Science and Engineering, National Institute of Technology, Patna, Bihar, India. His area of interest lies in Data Networks, Wireless Sensor Networks, E-learning Systems, Semantic Sensor Web and Security. 